\title{
On Dislocations in a Special Class of Generalized Elasticity
}

\author{
Markus Lazar ${ }^{\text {a,b,* }}$, Gérard A. Maugin a and Elias C. Aifantis ${ }^{\text {b,c }}$ \\ a Laboratoire de Modélisation en Mécanique, \\ Université Pierre et Marie Curie, \\ 4 Place Jussieu, Case 162, \\ F-75252 Paris Cedex 05, France \\ b Laboratory of Mechanics and Materials, Polytechnic School, \\ Aristotle University of Thessaloniki, P.O. Box 468, \\ 54124 Thessaloniki, Greece \\ c Center for the Mechanics of Material Instabilities and Manufacturing Processes, \\ Michigan Technological University, \\ Houghton, MI 49931, USA
}

September 13, 2018

\begin{abstract}
In this paper we consider and compare special classes of static theories of gradient elasticity, nonlocal elasticity, gradient micropolar elasticity and nonlocal micropolar elasticity with only one gradient coefficient. Equilibrium equations are discussed. The relationship between the gradient theory and the nonlocal theory is discussed for elasticity as well as for micropolar elasticity. Nonsingular solutions for the elastic fields of screw and edge dislocations are given. Both the elastic deformation (distortion, strain, bend-twist) and the force and couple stress tensors do not possess any singularity unlike 'classical' theories.
\end{abstract}

\section{Introduction}

The aim of this paper is to give an overview of a special class of generalizations of elasticity. In classical continuum mechanics, the elastic continuum is viewed as a collection of particles which have only three translational degrees of freedom. The particles are taken without structure and are idealized as point masses.

The first attempts to modify the theory of elasticity were done by taking rotational degrees of freedom in addition to the translational ones. In such media, which are called Cosserat (or micropolar) media, every material particle is considered as a rigid volume element. Each particle has six independent degrees of freedom: 3 micro-rotational and 3 translational ones of the center. Thus, a micropolar continuum can be described by geometrical points to which a micro-rotation vector is attached. Accordingly, a particle is identified by its position vector and its micro-rotation vector.

*Corresponding author. E-mail address: lazar@lmm.jussieu.fr (M. Lazar). 


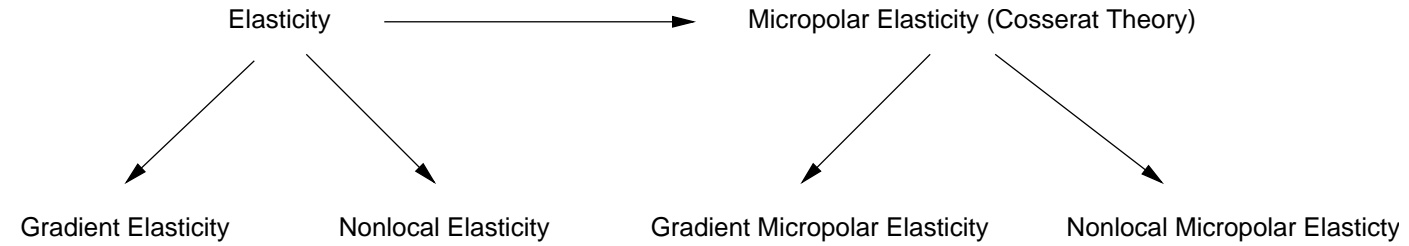

Figure 1: Overview of generalizations of elasticity

For the calculation of stresses, strains and distortions produced by defects (dislocations, disclinations, cracks) such theories break down near the defect lines because they are not valid at very small distances. For instance, the elastic stress field of a dislocation has a $1 / r$-singularity and the elastic couple stress field has a $1 / r^{2}$-singularity.

Thus, generalized continuum theories which are able to explain the material behaviour on the nanoscale are needed. Such theories can build bridges between continuum and atomic physics. On the other hand, the singularities in the elastic fields of defects should disappear in a theory which is also valid in the defect core region. The following question arises: What kind of generalized elasticity theories are able to do that?

An extension of elasticity theory which goes into another direction than the Cosserat theory is the theory of nonlocal elasticity. The concept of nonlocal elasticity was originally proposed by Kröner and Datta [1,2], Edelen and Eringen [3], Kunin [4] and some others. Nonlocal theories were introduced to explain the material behaviour on the nanoscale (e.g., in the core of defects). Such a theory considers the inner structure of materials and takes into account longrange (nonlocal) interactions. In a continuum theory, nonlocality arises due to the finite range of interaction between material points. The long range of interaction implies that the quantities such as stress and energy are functionals depending on the motion of all points of the body. The nonlocal kernel, which appears in the nonlocal constitutive relation between the stress and strain fields, weights the elastic constants of the material. The kernel is supposed to model the details of the atomic interaction. The theory of nonlocal elasticity was successfully applied to the calculation of the stresses produced by defects (cracks, dislocations, disclinations). The main goal is the elimination of the classical unphysical singularities of the stress fields [5-14]. Thus the stress fields are smooth even in the core region. In addition, nonlocal elasticity leads to a natural fracture criterion which is based on the maximum stress hypothesis $[9,10]$. So, materials will fracture when the calculated maximum stress reaches the cohesive stress of atomic bonds. However, the strain field singularities are still present in nonlocal elasticity.

Another extension of the classical theory of elasticity is called strain gradient elasticity and is very close to nonlocal elasticity. The physical motivation to introduce gradient theories was given by Kröner $[1,15]$ in the early sixties. The strain gradient theories extend the classical elasticity with additional strain gradient terms. Due to the gradients, they must contain additional material constants with the dimension of a length, and hyperstresses appear. The hyperstress tensor is a higher order stress tensor given in terms of strain gradient terms. In particular, early theories of isotropic, higher-order gradient, linear elasticity were developed essentially by Mindlin [16-18], Green and Rivlin [19,20]. Such gradient theories contain strain gradient terms and no rotation vector. In this way, they are different from theories with rotational degrees of freedom (Cosserat theory, micropolar elasticity). Only hyperstresses such as double or triple stresses appear in strain gradient theories [17]. Gradient elasticity was used to calculate the stress and strain fields produced by dislocations and disclinations [21-23]. The gradient elasticity solutions have no singularity in both the stress and the strain fields. On the other hand, in first gradient elasticity the double stresses still have singularities at the defect line [23]. We notice that in the special gradient elasticity used by Gutkin and Aifanis [24-26] the stresses are 
still singular. They only eliminated the strain singularities. Also, the gradient theories latter developed and used by Gutkin and Aifantis [21,22,27] and the gradient theory by Lazar and Maugin [23] are slightly different. Gutkin and Aifantis used two different gradient coefficients and Lazar and Maugin used only one gradient coefficient.

Eringen $[10,28]$ introduced a theory of nonlocal micropolar elasticity (or nonlocal Cosserat theory). In this theory the couple stress also has a nonlocal constitutive relation in addition to the asymmetric force stress tensor. By means of nonlocal micropolar elasticity, the singularities in both the force stresses and couple stresses can be eliminated [29,30]. But, the micropolar distortion and wryness still possess singularities. This was one motivation for Lazar and Maugin to introduce a gradient micropolar elasticity [31,32]. The gradient micropolar elasticity solutions eliminate these singularities at the defect line.

Thus, in this paper we only consider various generalizations of classical linear elasticity and not physically nonlinear elasticity. Nevertheless, physically nonlinear elasticity is an alternative way to eliminate classical singularities at the dislocation line (see, e.g., [33]). But nonlinear elasticity is still scale-free and no internal lengths appear.

The aim of this paper is to give an overview of the solutions of dislocations in gradient elasticity, nonlocal elasticity, gradient micropolar elasticity and nonlocal micropolar elasticity of Helmholtz type. Gradient theory of Helmholtz type coincides with a special class of first order gradient theory. Nonlocal elasticity of Helmholtz type means that the nonlocal kernel is the Green function of the two-dimensional Helmholtz equation. We give the results for screw and edge dislocations. We consider only gradient theories and nonlocal theories with only one gradient coefficient and one nonlocal parameter, respectively.

\section{Generalized Elasticity of Helmholtz type}

\subsection{Gradient elasticity of first order}

In first gradient elasticity, the strain energy is assumed to depend only on the elastic strain $E_{i j}$ and on the first gradients of it $[17,18,23,34]$

$$
W=W\left(E_{i j}, \partial_{k} E_{i j}\right),
$$

where in the most general case of linear elasticity - the incompatible elasticity - the elastic strain is given by

$$
E_{i j}=\frac{1}{2}\left(\partial_{j} u_{i}+\partial_{i} u_{j}\right)-E_{i j}^{P} .
$$

Here $u_{i}$ is the displacement vector and $E_{i j}^{P}$ denotes the plastic strain. The elastic strain (2.2) is the symmetric part of the elastic distortion

$$
\beta_{i j}=\partial_{j} u_{i}-\beta_{i j}^{P}
$$

where $\beta_{i j}^{P}$ is the plastic distortion. As usual, the gradient of the displacement defines the total distortion

$$
\beta_{i j}^{T}=\partial_{j} u_{i} .
$$

With Eq. (2.2) the strain energy (2.1) may be rewritten in terms of gradients of the displacement and the plastic strain according to

$$
W=W\left(\partial_{(i} u_{j)}, \partial_{k} \partial_{(i} u_{j)}, E_{i j}^{P}, \partial_{k} E_{i j}^{P}\right) .
$$

In the theory of dislocations (see, e.g., [35]), the dislocation tensor is defined by

$$
\alpha_{i j}=\epsilon_{j k l} \partial_{k} \beta_{i l}=-\epsilon_{j k l} \partial_{k} \beta_{i l}^{P}
$$


as incompatibility condition and, on the other hand, the compatibility condition for the total distortion reads

$$
\epsilon_{j k l} \partial_{k} \beta_{i l}^{T}=0
$$

By differentiating Eq. (2.6) we obtain the translational Bianchi identity for $\alpha_{i j}$ :

$$
\partial_{j} \alpha_{i j}=0 .
$$

If the plastic strain and plastic distortion are zero, we have compatible elastic strain and distortion which are only given in terms of the gradient of the displacement and, thus, the dislocation tensor (2.6) must be zero. Such compatible strain gradient theories were investigated by Mindlin [16-18]. Because we are interested in dislocations which cause plastic strain, we must use an incompatible strain gradient theory [23].

In gradient elasticity of Helmholtz type, the following expression of the strain energy is postulated $[23,34]$

$$
W=\frac{1}{2} \sigma_{i j} E_{i j}+\frac{1}{2} \varepsilon^{2}\left(\partial_{k} \sigma_{i j}\right)\left(\partial_{k} E_{i j}\right),
$$

where the parameter $\varepsilon$ is called the gradient coefficient which has the dimension of a length. The pertinent stress tensors can be defined by taking the variation of $W$

$$
\begin{aligned}
\sigma_{i j} & :=\frac{\partial W}{\partial E_{i j}}, \\
\tau_{i j k} & :=\frac{\partial W}{\partial\left(\partial_{k} E_{i j}\right)}=\varepsilon^{2} \partial_{k} \sigma_{i j}
\end{aligned}
$$

with the Hooke law for an isotropic media

$$
\sigma_{i j}=\lambda \delta_{i j} E_{k k}+2 \mu E_{i j}
$$

where $\lambda$ and $\mu$ are the Lamé coefficients. For nonnegative strain energy, $W \geq 0$, one has

$$
3 \lambda+2 \mu \geq 0, \quad \mu \geq 0, \quad \varepsilon^{2} \geq 0 .
$$

The stress tensor $\sigma_{i j}=\sigma_{j i}$ is a Cauchy-like stress tensor and $\tau_{i j k}=\tau_{j i k}$ is the double stress tensor. Consequently, the double stress (2.11) is a simple gradient of the Cauchy stress. Thus, it is a higher-order stress tensor. Only $\varepsilon^{2}$ is a non-standard coefficient of the theory.

Further, for vanishing external body forces, the force equilibrium equation can be obtained from the principle of virtual work as (variation with respect to the displacement) [16]

$$
\partial_{j}\left(\sigma_{i j}-\partial_{k} \tau_{i j k}\right)=0
$$

and with (2.11) we find

$$
\left(1-\varepsilon^{2} \Delta\right) \partial_{j} \sigma_{i j}=0
$$

where $\Delta$ denotes the Laplacian. Finally, it is convenient to introduce a quantity, which is called the total stress tensor, and is defined as

$$
\stackrel{\circ}{\sigma}_{i j}=\sigma_{i j}-\partial_{k} \tau_{i j k}=\lambda \delta_{i j} E_{k k}+2 \mu E_{i j}-\varepsilon^{2}\left(\lambda \delta_{i j} \Delta E_{k k}+2 \mu \Delta E_{i j}\right) .
$$

With this definition Eq. (2.14) takes the form

$$
\partial_{j} \stackrel{\circ}{\sigma j}_{i j}=0 .
$$


If we rewrite Eq. (2.16), we obtain for every component of the Cauchy stress an inhomogeneous Helmholtz equation

$$
\left(1-\varepsilon^{2} \Delta\right) \sigma_{i j}=\stackrel{\circ}{\sigma}_{i j}
$$

The RHS of (2.18) is given in terms of the total stress tensor.

Using the inverse of Hooke's law for the stress $\sigma_{i j}$ and $\stackrel{\circ}{\sigma}_{i j}$, it follows that the elastic strain can be determined from the equation

$$
\left(1-\varepsilon^{2} \Delta\right) E_{i j}=\stackrel{\circ}{E}_{i j}
$$

where $\stackrel{\circ}{E}_{i j}$ is the classical strain tensor.

Using Eqs. (2.2) and (2.3), we obtain the coupled partial differential equation

$$
\left(1-\varepsilon^{2} \Delta\right)\left[\partial_{(i} u_{j)}-\beta_{(i j)}^{P}\right]=\partial_{(i} \stackrel{\circ}{u}_{j)}-\stackrel{\circ}{\beta}_{(i j)}^{P},
$$

where $\stackrel{\circ}{u}_{i}$ denotes the displacement field and $\stackrel{\circ}{\beta}_{i j}^{P}$ is the plastic distortion in classical defect theory (see, e.g., [36]). Thus, if the following equations are satisfied

$$
\begin{aligned}
& \left(1-\varepsilon^{2} \Delta\right) \beta_{i j}=\stackrel{\circ}{\beta}_{i j}, \\
& \left(1-\varepsilon^{2} \Delta\right) \beta_{i j}^{P}=\stackrel{\circ}{\beta}_{i j}^{P},
\end{aligned}
$$

the equation for the displacement field,

$$
\left(1-\varepsilon^{2} \Delta\right) u_{i}=\stackrel{\circ}{u_{i}},
$$

is valid for the incompatible case. Thus, for defects (dislocations, disclinations) the inhomogeneous parts of Eqs. (2.22) and (2.23) are fields with discontinuities. The discontinuity of the displacement field of a defect is usually represented by a branch cut in order to make the multivalued part to a single-valued one. Thus this discontinuity is for mathematical convenience only. This can be accepted because the displacement and the plastic distortion are not physical state variables. Therefore, the displacement and the plastic distortion depend on the choice of the branch cut. For that reason, they are not unique.

From Eqs. (2.6), (2.21) and (2.22) we obtain

$$
\left(1-\varepsilon^{2} \Delta\right) \alpha_{i j}=\stackrel{\circ}{\alpha}_{i j}
$$

where $\stackrel{\circ}{\alpha}_{i j}$ is the classical dislocation density tensor. For a straight dislocation it has the form

$$
\stackrel{\circ}{\alpha}_{i j}=b_{i} \otimes n_{j} \delta(x) \delta(y)
$$

where $b_{i}$ and $n_{j}$ denote the Burgers vector and the direction of the dislocation line, respectively. Then $\alpha_{i j}$ is the two-dimensional Green function of (2.24) and reads

$$
\alpha_{i j}=\frac{1}{2 \pi \varepsilon^{2}} b_{i} \otimes n_{j} K_{0}(r / \varepsilon)
$$

where $r=\sqrt{x^{2}+y^{2}}$ and $K_{n}$ is the modified Bessel function of the second kind and $n=0,1, \ldots$ denotes the order of this function.

The limit $\varepsilon \rightarrow 0$ is the limit from gradient elasticity theory to classical theory of elasticity. 


\subsection{Nonlocal Elasticity}

The basic equations of linear, isotropic, nonlocal elastic solids, for the static case with vanishing body force, are $[5,6,8,10]$

$$
\begin{aligned}
& \partial_{j} \sigma_{i j}=0, \\
& \sigma_{i j}(r)=\int_{V} \alpha\left(r-r^{\prime}\right) \sigma_{i j}^{(\mathrm{cl})}\left(r^{\prime}\right) \mathrm{d} v\left(r^{\prime}\right), \\
& \sigma_{i j}^{(\mathrm{cl})}=\lambda \delta_{i j} \epsilon_{k k}^{(\mathrm{cl})}+2 \mu \epsilon_{i j}^{(\mathrm{cl})} .
\end{aligned}
$$

Here $\epsilon_{i j}^{(\mathrm{cl})}$ is the classical strain tensor, $\sigma_{i j}^{(\mathrm{cl})}$, and $\sigma_{i j}$ are the classical and nonlocal stress tensors, respectively. In addition, $\lambda$ and $\mu$ are the Lamé constants and $\alpha(r)$ is the 'attenuation function' called nonlocal kernel. It is important to note that the local material coefficients in Eqs. (2.29) are the same which appear in (2.12).

Eringen and Ari [7] found for the two-dimensional case that an excellent match of phonon dispersion was obtained, in the entire Brillouin zone, by

$$
\alpha\left(r-r^{\prime}\right)=\frac{1}{2 \pi \varepsilon^{2}} K_{0}\left(\sqrt{\left(x-x^{\prime}\right)^{2}+\left(y-y^{\prime}\right)^{2}} / \varepsilon\right), \quad \varepsilon \geq 0 .
$$

$\varepsilon$ is called the parameter of nonlocality. It is interesting to note that the nonlocal kernel (2.30) is the Green function for the two-dimensional Helmholtz equation

$$
\left(1-\varepsilon^{2} \Delta\right) \alpha(r)=\delta(x) \delta(y) .
$$

Thus, we call (2.30) nonlocal kernel of Helmholtz-type. In this way, we deduce Eringen's socalled nonlocal constitutive relation for a linear, isotropic solid with Green's function (2.30) as the nonlocal kernel. This kernel (2.30) has its maximum at $r=r^{\prime}$ and describes the nonlocal interaction. The normalization condition for the nonlocal kernel is given by

$$
\int_{V} \alpha\left(r-r^{\prime}\right) \mathrm{d} v\left(r^{\prime}\right)=1
$$

In the classical limit $(\varepsilon \rightarrow 0)$, it becomes the two-dimensional Dirac delta function

$$
\lim _{\varepsilon \rightarrow 0} \alpha\left(r-r^{\prime}\right)=\delta\left(x-x^{\prime}\right) \delta\left(y-y^{\prime}\right) .
$$

In this limit, Eq. (2.28) gives the classical expression. We notice that Eringen $[8,10]$ found the two-dimensional kernel (2.30) by giving the best match with the Born-Kármán model of the atomic lattice dynamics and the atomistic dispersion curves. He used the choice $e_{0}=0.39$ for the length, $\varepsilon=e_{0} a$, where $a$ is an internal length (e.g. atomic lattice parameter) and $e_{0}$ is a constant appropriate to each material.

Applying the Helmholtz operator $\left(1-\varepsilon^{2} \Delta\right)$ to $(2.28)$, we find the following inhomogeneous Helmholtz equation

$$
\left(1-\varepsilon^{2} \Delta\right) \sigma_{i j}=\sigma_{i j}^{(\mathrm{cl})},
$$

where $\sigma_{i j}^{(\mathrm{cl})}$ is the stress tensor obtained for the same traction boundary-value problem within the 'classical' theory. The factor $\varepsilon$ has the physical dimension of a length and it, therefore, defines an internal characteristic length.

It is obvious that a 'nonlocal' strain tensor does not appear in nonlocal elasticity. Thus, the strain and the displacement fields are the classical ones in Eringen's theory of nonlocal elasticity. The same is true for the double stresses which occur in gradient elasticity. If we identify $\sigma_{i j}^{(\mathrm{cl})}=\stackrel{\circ}{\sigma}_{i j}$, Eqs. (2.18) and (2.34) coincide and the stress $\sigma_{i j}$ also fulfills $\partial_{j} \sigma_{i j}=0$ in gradient elasticity. Then the total stress tensor is equal to the classical stress tensor. Only, the stress tensor $\sigma_{i j}$ is the unknown field which must be found. 


\subsection{Screw Dislocation}

We begin with the simplest case of a defect - the straight screw dislocation. First, we need the classical quantities because they act as inhomogeneous parts in gradient elasticity as well in nonlocal elasticity. The classical stress is given by

$$
\stackrel{\circ}{\sigma}_{z \varphi}=\partial_{r} \stackrel{\circ}{F}=\frac{\mu b_{z}}{2 \pi r},
$$

with the Prandtl stress function

$$
\stackrel{\circ}{F}=\frac{\mu b_{z}}{2 \pi} \ln r .
$$

The modified stress can be expressed in terms of a new stress function $F$ as follows

$$
\sigma_{z \varphi}=\partial_{r} F
$$

For a screw dislocation Eq. (2.18) takes the form

$$
\left(1-\varepsilon^{2} \Delta\right) F=\frac{\mu b_{z}}{2 \pi} \ln r .
$$

The nonsingular solution of the modified stress function for a screw dislocation is given by

$$
F=\frac{\mu b_{z}}{2 \pi}\left\{\ln r+K_{0}(r / \varepsilon)\right\},
$$

which is a superposition of the Prandtl stress function and a gradient part depending on $\varepsilon$. Thus, it is the two-dimensional Green function of the following partial differential equation of fourth order (Helmholtz Laplace equation)

$$
\left(1-\varepsilon^{2} \Delta\right) \Delta F=\mu b_{z} \delta(x) \delta(y) .
$$

Because of the fact that (2.39) satisfies the inhomogeneous Helmholtz equation in which the Prandtl stress function is the inhomogeneous part, one might call (2.39) the Helmholtz-modified Prandtl stress function.

Using Eqs. (2.37) and (2.39), the stress field is given by

$$
\sigma_{z \varphi}=\frac{\mu b_{z}}{2 \pi} \frac{1}{r}\left\{1-\frac{r}{\varepsilon} K_{1}(r / \varepsilon)\right\} .
$$

Unlike the classical result, it does not possess any singularity at $r=0$. It has a maximum $\sigma_{z \varphi} \simeq 0.399 \mu b_{z} /[2 \pi \varepsilon]$ at about $r \simeq 1.114 \varepsilon$. The plot of the stress versus $r / \varepsilon$ is shown in Fig. 2. The stress (2.41) is also the solution of Eq. (2.34) in nonlocal elasticity. In nonlocal elasticity this solution was found by Eringen [8]. It is interesting to notice that a solution of a screw dislocation in nonlocal elasticity with a nonlocal kernel which is the Green function of a diffusion-like equation was earlier given by Eringen [5].

By means of the inverse of Hooke's law, the elastic strain is given by

$$
E_{z \varphi}=\frac{b_{z}}{4 \pi} \frac{1}{r}\left\{1-\frac{r}{\varepsilon} K_{1}(r / \varepsilon)\right\},
$$

which is nonsingular at the dislocation line and has its maximum $E_{z \varphi} \simeq 0.399 b_{z} /[2 \pi \varepsilon]$ at $r \simeq 1.114 \varepsilon$. The components of stresses and strains in Cartesian coordinates are given in Refs. [21-24,37]. 


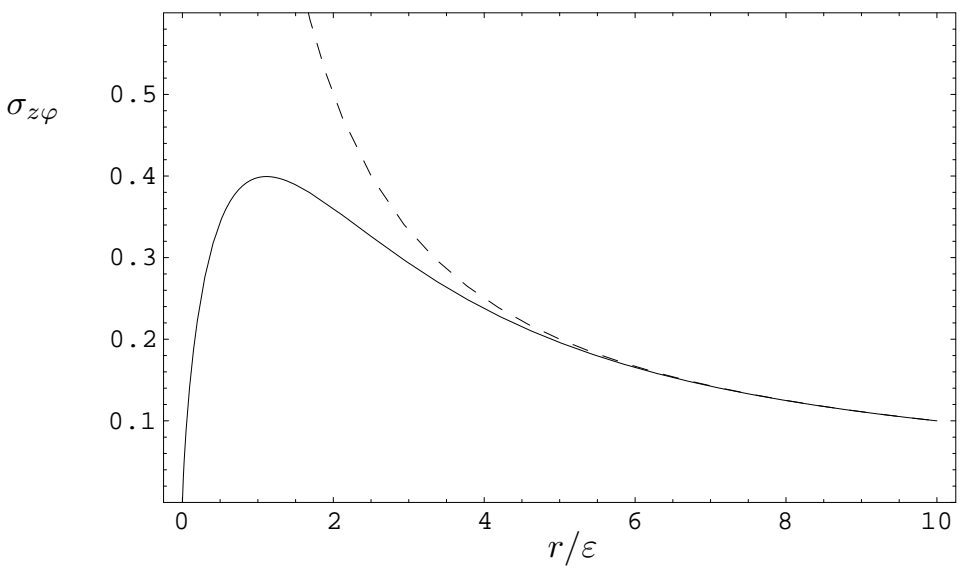

Figure 2: The stress $\sigma_{z \varphi}$ is given in units of $\mu b_{z} /[2 \pi \varepsilon]$. The dashed curve represents the classical result.

\subsection{Edge Dislocation}

The components of the classical stress of a straight edge dislocation with Burgers vector along the $x$-direction read in cylindrical coordinates

$$
\stackrel{\circ}{\sigma}_{r r}=\frac{1}{r} \partial_{r} \stackrel{\circ}{f}+\frac{1}{r^{2}} \partial_{\varphi \varphi}^{2} \stackrel{\circ}{f}, \quad \stackrel{\circ}{\sigma}_{\varphi \varphi}=\partial_{r r}^{2} \stackrel{\circ}{f}, \quad \stackrel{\circ}{\sigma}_{r \varphi}=-\partial_{r}\left(\frac{1}{r} \partial_{\varphi} f\right), \quad \stackrel{\circ}{\sigma}_{z z}=\nu\left(\stackrel{\circ}{\sigma}_{r r}+\stackrel{\circ}{\sigma}_{\varphi \varphi}\right),
$$

where the Airy stress function is given by

$$
\stackrel{\circ}{f}=-\frac{\mu b_{x}}{2 \pi(1-\nu)} \sin \varphi(r \ln r) .
$$

For the modified stress of an edge dislocation, the stresses are given in terms of a stress function $f$ similar to $(2.43)$

$$
\sigma_{r r}=\frac{1}{r} \partial_{r} f+\frac{1}{r^{2}} \partial_{\varphi \varphi}^{2} f, \quad \sigma_{\varphi \varphi}=\partial_{r r}^{2} f, \quad \sigma_{r \varphi}=-\partial_{r}\left(\frac{1}{r} \partial_{\varphi} f\right), \quad \sigma_{z z}=\nu\left(\sigma_{r r}+\sigma_{\varphi \varphi}\right) .
$$

For an edge dislocation (2.18) has the following form

$$
\left(1-\varepsilon^{2} \Delta\right) f=-\frac{\mu b_{x}}{2 \pi(1-\nu)} \sin \varphi(r \ln r),
$$

with the Airy function as inhomogeneous part. The nonsingular solution of (2.46) is given by $[14]$

$$
f=-\frac{\mu b_{x}}{2 \pi(1-\nu)} \sin \varphi\left\{r \ln r+\frac{2 \varepsilon^{2}}{r}\left(1-\frac{r}{\varepsilon} K_{1}(r / \varepsilon)\right)\right\} .
$$

It is a superposition of the Airy stress function and a gradient part. We may call the stress function (2.47) a Helmholtz-modified Airy stress function since it fulfills (Helmholtz bi-Laplace equation)

$$
\left(1-\varepsilon^{2} \Delta\right) \Delta \Delta f=-\frac{2 \mu b_{x}}{(1-\nu)} \partial_{y} \delta(x) \delta(y)
$$


Thus, it is a Green function of this partial differential equation of sixth order.

By means of Eqs. (2.45) and (2.47), the stress field of an edge dislocation is calculated as

$$
\begin{aligned}
\sigma_{r r} & =-\frac{\mu b_{x}}{2 \pi(1-\nu)} \frac{\sin \varphi}{r}\left\{1-\frac{4 \varepsilon^{2}}{r^{2}}+2 K_{2}(r / \varepsilon)\right\}, \\
\sigma_{r \varphi} & =\frac{\mu b_{x}}{2 \pi(1-\nu)} \frac{\cos \varphi}{r}\left\{1-\frac{4 \varepsilon^{2}}{r^{2}}+2 K_{2}(r / \varepsilon)\right\}, \\
\sigma_{\varphi \varphi} & =-\frac{\mu b_{x}}{2 \pi(1-\nu)} \frac{\sin \varphi}{r}\left\{1+\frac{4 \varepsilon^{2}}{r^{2}}-2 K_{2}(r / \varepsilon)-2 \frac{r}{\varepsilon} K_{1}(r / \varepsilon)\right\}, \\
\sigma_{z z} & =-\frac{\mu b_{x} \nu}{\pi(1-\nu)} \frac{\sin \varphi}{r}\left\{1-\frac{r}{\varepsilon} K_{1}(r / \varepsilon)\right\},
\end{aligned}
$$

and the trace of the stress tensor reads

$$
\sigma_{k k}=-\frac{\mu b_{x}(1+\nu)}{\pi(1-\nu)} \frac{\sin \varphi}{r}\left\{1-\frac{r}{\varepsilon} K_{1}(r / \varepsilon)\right\}
$$

The stress is plotted over $r / \varepsilon$ in Fig. 3. Based on the present solution (2.49)-(2.53) we observe that the stress fields are not singular at $r=0$. In fact, they are zero at $r=0$. The radial extremum values are: $\sigma_{r r} \simeq-0.259 \mu b_{x} /[2 \pi(1-\nu) \varepsilon] \sin \varphi$ at $r \simeq 1.494 \varepsilon, \sigma_{r \varphi} \simeq$ $0.259 \mu b_{x} /[2 \pi(1-\nu) \varepsilon] \cos \varphi$ at $r \simeq 1.494 \varepsilon, \sigma_{\varphi \varphi} \simeq-0.546 \mu b_{x} /[2 \pi(1-\nu) \varepsilon] \sin \varphi$ at $r \simeq 0.546 \varepsilon$, $\sigma_{z z} \simeq-0.399 \mu b_{x} \nu /[\pi(1-\nu) \varepsilon] \sin \varphi$ at $r \simeq 1.114 \varepsilon$ and $\sigma_{k k} \simeq-0.399 \mu b_{x}(1+\nu) /[\pi(1-\nu) \varepsilon] \sin \varphi$ at $r \simeq 1.114 \varepsilon$. We note that the stresses (2.49)-(2.53) are also solution of Eq. (2.34) in the nonlocal elasticity. The solution of an edge dislocation given by Eringen [6] does not correspond to the nonlocal kernel (2.30). It is the solution in nonlocal elasticity with the nonlocal kernel which is Green's function of a diffusion-like equation. Therefore, it is different.

The elastic strain of a straight edge dislocation is given by

$$
\begin{aligned}
& E_{r r}=-\frac{b_{x}}{4 \pi(1-\nu)} \frac{\sin \varphi}{r}\left\{(1-2 \nu)-\frac{4 \varepsilon^{2}}{r^{2}}+2 K_{2}(r / \varepsilon)+2 \nu \frac{r}{\varepsilon} K_{1}(r / \varepsilon)\right\}, \\
& E_{r \varphi}=\frac{b_{x}}{4 \pi(1-\nu)} \frac{\cos \varphi}{r}\left\{1-\frac{4 \varepsilon^{2}}{r^{2}}+2 K_{2}(r / \varepsilon)\right\}, \\
& E_{\varphi \varphi}=-\frac{b_{x}}{4 \pi(1-\nu)} \frac{\sin \varphi}{r}\left\{(1-2 \nu)+\frac{4 \varepsilon^{2}}{r^{2}}-2 K_{2}(r / \varepsilon)-2(1-\nu) \frac{r}{\varepsilon} K_{1}(r / \varepsilon)\right\} .
\end{aligned}
$$

The dilatation reads

$$
E_{k k}=-\frac{b_{x}(1-2 \nu)}{2 \pi(1-\nu)} \frac{\sin \varphi}{r}\left\{1-\frac{r}{\varepsilon} K_{1}(r / \varepsilon)\right\}
$$

Again the strain fields (2.54)-(2.57) are not singular at $r=0$. They are zero at the dislocation line. They have the following radial extremum values $(\nu=0.3)$ : The strain $E_{r r}$ has two extremum values of opposite signs (see Fig. 4a), namely, $E_{r r} \simeq 0.010 b_{x} /[4 \pi(1-\nu) \varepsilon] \sin \varphi$ at $r \simeq 0.218 \varepsilon$ and $E_{r r} \simeq-0.054 b_{x} /[4 \pi(1-\nu) \varepsilon] \sin \varphi$ at $r \simeq 4.130 \varepsilon$. In addition, it is equal to zero at $r \simeq 0.677 \varepsilon$. On the other hand, $E_{r \varphi} \simeq 0.259 b_{x} /[4 \pi(1-\nu) \varepsilon] \cos \varphi$ at $r \simeq 1.494 \varepsilon$, $E_{\varphi \varphi} \simeq-0.308 b_{x} /[4 \pi(1-\nu) \varepsilon] \sin \varphi$ at $r \simeq 0.922 \varepsilon$ (see Fig. 4b) and $E_{k k} \simeq-0.399 \mu b_{x}(1-$ $2 \nu) /[2 \pi(1-\nu) \varepsilon] \sin \varphi$ at $r \simeq 1.114 \varepsilon$. Unlike the classical solution, $E_{r r}$ is significantly smaller than $E_{\varphi \varphi}$ in the dislocation core region. The components of the stress and strain tensors in Cartesian coordinates can be found in Refs. [14,21-23, 25, 37]. 

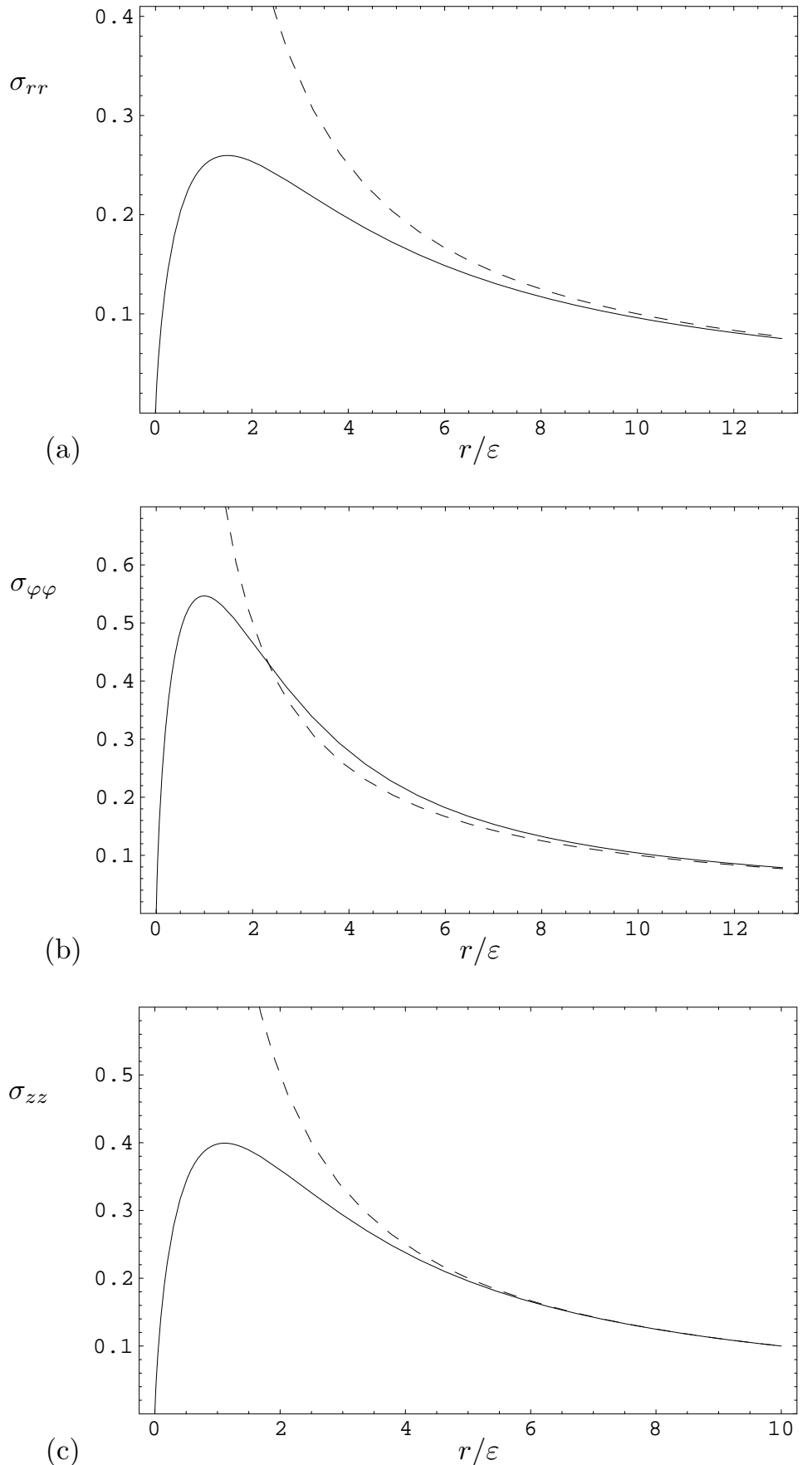

Figure 3: The components of stress: (a) $\sigma_{r r}$ and (b) $\sigma_{\varphi \varphi}$ are given in units of $\mu b_{z} /[2 \pi(1-\nu) \varepsilon]$ and (c) $\sigma_{z z}$ is given in units of $\mu b_{z} \nu /[\pi(1-\nu) \varepsilon]$ for $\varphi=3 \pi / 2$. The dashed curves represent the classical components. 

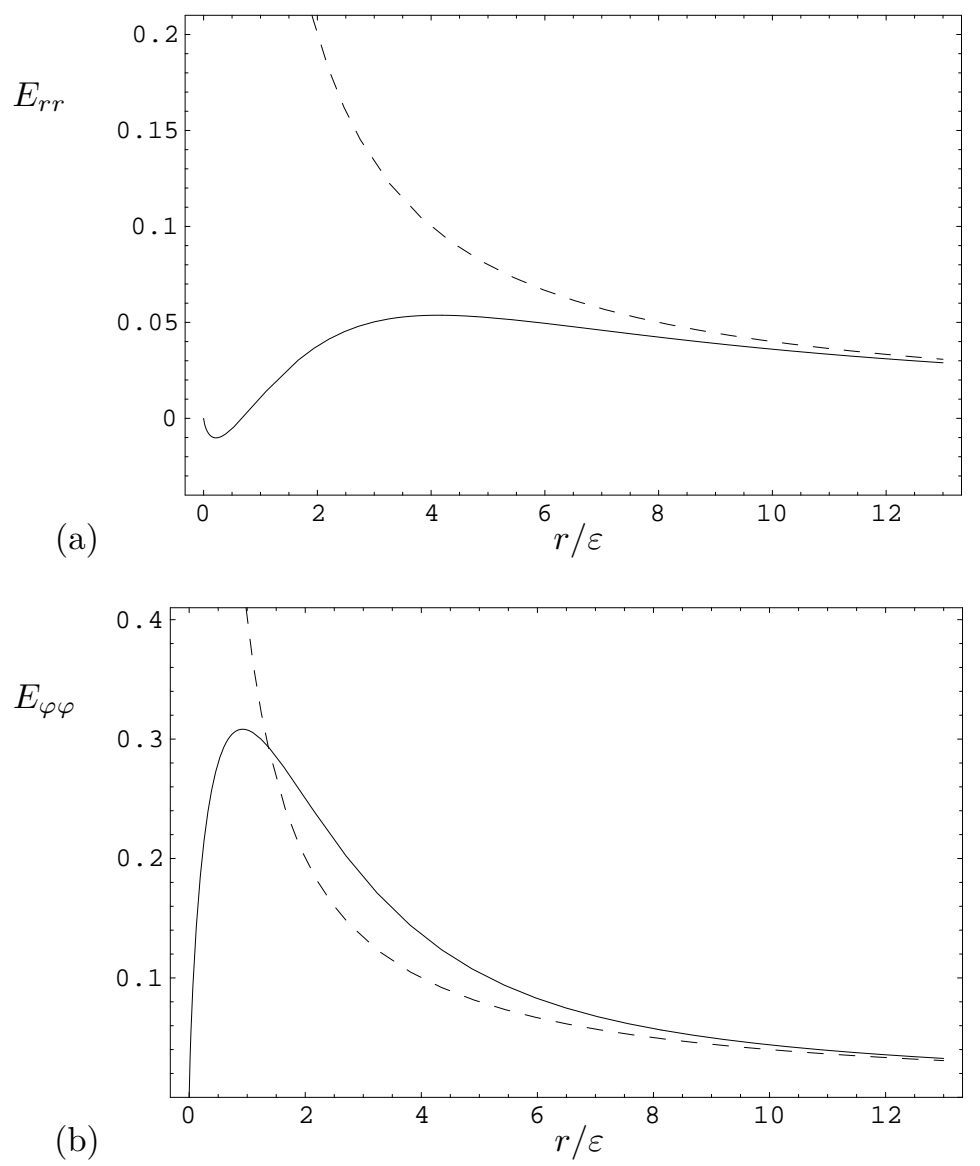

Figure 4: The components of strain: (a) $E_{r r}$ and (b) $E_{\varphi \varphi}$ are given in units of $b_{z} /[4 \pi(1-\nu) \varepsilon]$ for $\nu=0.3$ and $\varphi=3 \pi / 2$. The dashed curves represent the classical components.

\section{Generalized Micropolar Elasticity of Helmholtz type}

\subsection{Gradient Micropolar Elasticity of First Order}

In first gradient micropolar elasticity (or gradient Cosserat theory), the strain energy is assumed to depend only on the micropolar distortion $\gamma_{i j}$, the micropolar bend-twist $\kappa_{i j}$ and on the first gradients of them $[31,32]$

$$
W=W\left(\gamma_{i j}, \kappa_{i j}, \partial_{k} \gamma_{i j}, \partial_{k} \kappa_{i j}\right),
$$

where in the most general case - the incompatible micropolar elasticity - the elastic micropolar distortion is given by $[31,38]$

$$
\gamma_{i j}=\partial_{j} u_{i}+\epsilon_{i j k} \varphi_{k}-\gamma_{i j}^{P}
$$

Here $\varphi_{i}$ is the micro-rotation vector and $\gamma_{i j}^{P}$ denotes the plastic micropolar distortion. In addition, the elastic micropolar bend-twist (or wryness) reads [31,38]

$$
\kappa_{i j}=\partial_{j} \varphi_{i}-\kappa_{i j}^{P}
$$


where $\kappa_{i j}^{P}$ denotes the plastic micropolar bend-twist tensor. The total deformation quantities are given by

$$
\begin{aligned}
\gamma_{i j}^{T} & =\partial_{j} u_{i}+\epsilon_{i j k} \varphi_{k} \\
\kappa_{i j}^{T} & =\partial_{j} \varphi_{i} .
\end{aligned}
$$

For dislocation and disclination it is necessary to use the incompatible gradient theory because both defects cause plastic fields. Using Eqs. (3.2) and (3.3), the strain energy (3.1) might be written according to

$$
W=W\left(\partial_{j} u_{i}, \partial_{k} \partial_{j} u_{i}, \varphi_{i}, \partial_{j} \varphi_{i}, \partial_{k} \partial_{j} \varphi_{i}, \gamma_{i j}^{P}, \partial_{k} \gamma_{i j}^{P}, \kappa_{i j}^{P}, \partial_{k} \kappa_{i j}^{P}\right) .
$$

Thus, the expression (3.6) contains gradients of the displacement and rotation vectors in addition to gradients of the plastic fields.

By means of the plastic fields and the incompatible elastic ones the dislocation and disclination densities can be calculated as $[31,38]$.

$$
\begin{aligned}
& \alpha_{i j}=\epsilon_{j k l}\left(\partial_{k} \gamma_{i l}-\epsilon_{i l m} \kappa_{m k}\right)=-\epsilon_{j k l}\left(\partial_{k} \gamma_{i l}^{P}-\epsilon_{i l m} \kappa_{m k}^{P}\right), \\
& \Theta_{i j}=\epsilon_{j k l} \partial_{k} \kappa_{i l}=-\epsilon_{j k l} \partial_{k} \kappa_{i l}^{P} .
\end{aligned}
$$

By differentiating Eq. (3.7) and Eq. (3.8) we obtain the translational and rotational Bianchi identities for the dislocation and disclination density tensors in the theory of micropolar elasticity:

$$
\begin{aligned}
\partial_{j} \alpha_{i j}-\epsilon_{i j k} \Theta_{j k} & =0, \\
\partial_{j} \Theta_{i j} & =0 .
\end{aligned}
$$

The compatibility conditions are

$$
\begin{aligned}
\epsilon_{j k l}\left(\partial_{k} \gamma_{i l}^{T}-\epsilon_{i l m} \kappa_{m k}^{T}\right) & =0, \\
\epsilon_{j k l} \partial_{k} \kappa_{i l}^{T} & =0 .
\end{aligned}
$$

When disclinations are absent, the disclination density tensor $\Theta_{i j}$ and the plastic micropolar bend-twist tensor $\kappa_{i j}^{P}$ must be zero and we have $\kappa_{i j}=\partial_{j} \varphi_{i}$ and $\gamma_{i j}^{P}=\beta_{i j}^{P}$. Then Eq. (3.8) is the compatibility condition for $\kappa_{i j}$. The elastic micropolar distortion (3.2) may be rewritten in the form

$$
\gamma_{i j}=\beta_{i j}+\epsilon_{i j k} \varphi_{k}
$$

Then the dislocation density tensor simplifies to

$$
\alpha_{i j}=\epsilon_{j k l} \partial_{k} \beta_{i l}=-\epsilon_{j k l} \partial_{k} \beta_{i l}^{P} .
$$

In gradient micropolar elasticity of Helmholtz type the strain energy, $W$, is assumed to be given as $[31,32]$

$$
W=\frac{1}{2} \sigma_{i j} \gamma_{i j}+\frac{1}{2} \mu_{i j} \kappa_{i j}+\frac{1}{2} \varepsilon^{2}\left(\partial_{k} \sigma_{i j}\right)\left(\partial_{k} \gamma_{i j}\right)+\frac{1}{2} \varepsilon^{2}\left(\partial_{k} \mu_{i j}\right)\left(\partial_{k} \kappa_{i j}\right),
$$

where $\kappa$ is the only one gradient coefficient. By means of the strain energy (3.1), the asymmetric 
stresses are defined by

$$
\begin{aligned}
\sigma_{i j} & :=\frac{\partial W}{\partial \gamma_{i j}} \\
\mu_{i j} & :=\frac{\partial W}{\partial \kappa_{i j}}, \\
\tau_{i j k} & :=\frac{\partial W}{\partial\left(\partial_{k} \gamma_{i j}\right)}=\varepsilon^{2} \partial_{k} \sigma_{i j}, \\
\lambda_{i j k} & :=\frac{\partial W}{\partial\left(\partial_{k} \kappa_{i j}\right)}=\varepsilon^{2} \partial_{k} \mu_{i j} .
\end{aligned}
$$

Here $\sigma_{i j}$ and $\mu_{i j}$ are the force stress tensor and couple stress tensor, respectively. The higher order stresses $\tau_{i j k}$ and $\lambda_{i j k}$ are called double force stress and double couple stress, respectively.

The constitutive relations for isotropic micropolar elasticity (see, e.g., $[10,39,40]$ ) have the form

$$
\begin{aligned}
& \sigma_{i j}=\lambda \delta_{i j} \gamma_{k k}+(\mu+\eta) \gamma_{i j}+(\mu-\eta) \gamma_{j i}, \\
& \mu_{i j}=\alpha \delta_{i j} \kappa_{k k}+(\beta+\gamma) \kappa_{i j}+(\beta-\gamma) \kappa_{j i} .
\end{aligned}
$$

Here $\lambda, \mu, \eta, \alpha, \beta$ and $\gamma$ are the 6 material constants of the isotropic Cosserat continuum. For nonnegative strain energy, $W \geq 0$, we have

$$
\begin{aligned}
& 3 \lambda+2 \mu \geq 0, \quad \mu \geq 0, \quad \eta \geq 0, \\
& 3 \alpha+2 \beta \geq 0, \quad \beta \geq 0, \quad \gamma \geq 0
\end{aligned}
$$

and

$$
\varepsilon^{2} \geq 0
$$

Using the six material constants of a Cosserat continuum (micropolar medium), two characteristic lengths $l$ and $h$ can be defined by $[39,40]$

$$
l^{2}=\frac{(\mu+\eta)(\beta+\gamma)}{4 \mu \eta}, \quad h^{2}=\frac{\alpha+2 \beta}{4 \eta},
$$

which are Cosserat intrinsic lengths. The above equations (3.20) and (3.21) may be expressed in terms of stresses

$$
\begin{aligned}
& \gamma_{i j}=\lambda^{\prime} \delta_{i j} \sigma_{k k}+\left(\mu^{\prime}+\eta^{\prime}\right) \sigma_{i j}+\left(\mu^{\prime}-\eta^{\prime}\right) \sigma_{j i}, \\
& \kappa_{i j}=\alpha^{\prime} \delta_{i j} \mu_{k k}+\left(\beta^{\prime}+\gamma^{\prime}\right) \mu_{i j}+\left(\beta^{\prime}-\gamma^{\prime}\right) \mu_{j i} .
\end{aligned}
$$

Here

$$
\begin{aligned}
& 2 \mu^{\prime}=\frac{1}{2 \mu}, \quad 2 \eta^{\prime}=\frac{1}{2 \eta}, \quad 2 \beta^{\prime}=\frac{1}{2 \beta}, \quad 2 \gamma^{\prime}=\frac{1}{2 \gamma}, \\
& \lambda^{\prime}=-\frac{\lambda}{2 \mu(3 \lambda+2 \mu)}, \quad \alpha^{\prime}=-\frac{\alpha}{2 \beta(3 \alpha+2 \beta)} .
\end{aligned}
$$

After variation of (3.1) with respect to the displacement $u_{i}$ and the rotation vector $\varphi_{i}$, the following equilibrium conditions follow

$$
\begin{aligned}
& \partial_{j}\left(\sigma_{i j}-\partial_{k} \tau_{i j k}\right)=0, \\
& \partial_{j}\left(\mu_{i j}-\partial_{k} \lambda_{i j k}\right)-\epsilon_{i j k}\left(\sigma_{j k}-\partial_{l} \tau_{j k l}\right)=0 .
\end{aligned}
$$


We may define the total stress and total couple stress tensors

$$
\begin{aligned}
& \stackrel{\circ}{\sigma}_{i j}=\sigma_{i j}-\partial_{k} \tau_{i j k}, \\
& \stackrel{\circ}{\mu}_{i j}=\mu_{i j}-\partial_{k} \lambda_{i j k} .
\end{aligned}
$$

So, Eqs. (3.29) and (3.30) take the form

$$
\begin{aligned}
& \partial_{j} \stackrel{\circ}{\sigma}_{i j}=0, \\
& \partial_{j} \stackrel{\circ}{\mu}_{i j}-\epsilon_{i j k} \stackrel{\circ}{\sigma}_{j k}=0
\end{aligned}
$$

or in terms of $\sigma_{i j}$ and $\mu_{i j}$

$$
\begin{aligned}
& \left(1-\varepsilon^{2} \Delta\right) \partial_{j} \sigma_{i j}=0, \\
& \left(1-\varepsilon^{2} \Delta\right)\left(\partial_{j} \mu_{i j}-\epsilon_{i j k} \sigma_{j k}\right)=0 .
\end{aligned}
$$

From Eqs. (3.31), (3.32), (3.18) and (3.19) the force stress tensor $\sigma_{i j}$ and couple stress tensor $\mu_{i j}$ satisfy the inhomogeneous Helmholtz equations

$$
\begin{aligned}
& \left(1-\varepsilon^{2} \Delta\right) \sigma_{i j}=\stackrel{\circ}{\sigma}_{i j}, \\
& \left(1-\varepsilon^{2} \Delta\right) \mu_{i j}=\stackrel{\circ}{\mu}_{i j},
\end{aligned}
$$

where the RHS are given in terms of total stress and couple stress tensors.

Using Eqs. (3.25) and (3.26), the inverse of Hooke's law for the stress $\sigma_{i j}$ and $\stackrel{\circ}{\sigma}_{i j}$, it follows that the elastic strain can be determined from the equation

$$
\begin{aligned}
& \left(1-\varepsilon^{2} \Delta\right) \gamma_{i j}=\stackrel{\circ}{\gamma}_{i j}, \\
& \left(1-\varepsilon^{2} \Delta\right) \kappa_{i j}=\stackrel{\circ}{\kappa}_{i j},
\end{aligned}
$$

where $\stackrel{\circ}{\gamma}_{i j}$ and $\stackrel{\circ}{\kappa}_{i j}$ are fields in 'classical' micropolar elasticity. These are coupled partial differential equations. In fact, substituting Eqs. (3.2) and (3.3) in (3.39) and (3.40), we obtain

$$
\begin{aligned}
& \left(1-\varepsilon^{2} \Delta\right)\left[\partial_{i} u_{j}+\epsilon_{i j k} \varphi_{k}-\gamma_{i j}^{P}\right]=\partial_{i} \stackrel{\circ}{u}_{j}+\epsilon_{i j k} \stackrel{\circ}{\varphi}_{k}-\stackrel{\circ}{\gamma}_{i j}^{P}, \\
& \left(1-\varepsilon^{2} \Delta\right)\left[\partial_{i} \varphi_{j}-\kappa_{i j}^{P}\right]=\partial_{i} \stackrel{\circ}{\varphi}_{j}-\stackrel{\circ}{\kappa}_{i j}^{P},
\end{aligned}
$$

where $\stackrel{\circ}{\varphi}_{i}$ denotes the rotation field, $\stackrel{\circ}{\gamma}_{i j}^{P}$ is the plastic micropolar distortion in defect theory (see, e.g., [38]). Thus, if and if only the following equations are fulfilled

$$
\begin{aligned}
& \left(1-\varepsilon^{2} \Delta\right) \gamma_{i j}^{P}=\stackrel{\circ}{\gamma}_{i j}^{P}, \\
& \left(1-\varepsilon^{2} \Delta\right) \kappa_{i j}^{P}=\stackrel{\circ}{\kappa}_{i j}^{P},
\end{aligned}
$$

the equations for the displacement and rotation fields,

$$
\begin{aligned}
\left(1-\varepsilon^{2} \Delta\right) u_{i} & =\stackrel{\circ}{u}_{i}, \\
\left(1-\varepsilon^{2} \Delta\right) \varphi_{i} & =\stackrel{\circ}{\varphi}_{i},
\end{aligned}
$$

are valid for the incompatible case. Since $\stackrel{P}{\kappa}_{i j}^{P}$ and $\kappa_{i j}^{P}$ are the plastic fields of disclinations, they are zero for dislocations. In this way, Eq. (3.46 follows directly from (3.42) for dislocations. Thus, in dislocation theory only the inhomogeneous parts of Eqs. (3.45) and (3.43) are fields with discontinuities. 
In addition, we obtain for defects in first gradient micropolar elasticity from Eqs. (3.7), (3.8), (3.39) and (3.40)

$$
\begin{aligned}
& \left(1-\varepsilon^{2} \Delta\right) \alpha_{i j}=\stackrel{\circ}{\alpha}_{i j}, \\
& \left(1-\varepsilon^{2} \Delta\right) \Theta_{i j}=\stackrel{\circ}{\Theta}_{i j},
\end{aligned}
$$

where $\stackrel{\circ}{\Theta}_{i j}$ is the classical disclination density tensor. For a straight disclination it reads

$$
\stackrel{\circ}{\Theta}_{i j}=\Omega_{i} \otimes n_{j} \delta(x) \delta(y),
$$

where $\Omega_{i}$ denotes the Frank vector. Consequently, the dislocation density and the disclination density tensors of a straight defect have in first gradient micropolar elasticity the following form

$$
\begin{aligned}
\alpha_{i j} & =\frac{1}{2 \pi \varepsilon^{2}} b_{i} \otimes n_{j} K_{0}(r / \varepsilon) \\
\Theta_{i j} & =\frac{1}{2 \pi \varepsilon^{2}} \Omega_{i} \otimes n_{j} K_{0}(r / \varepsilon) .
\end{aligned}
$$

In the limit $\varepsilon \rightarrow 0$, we recover micropolar elasticity. Thus, it is the 'classical' limit in gradient micropolar elasticity.

\section{$3.2 \quad$ Nonlocal Micropolar Elasticity}

The basic equations in the isotropic theory of nonlocal micropolar elasticity are given by $[10,28]$

$$
\begin{aligned}
& \partial_{j} \sigma_{i j}=0 \\
& \partial_{j} \mu_{i j}=\epsilon_{i j k} \sigma_{j k}=0, \\
& \sigma_{i j}(r)=\int_{V} \alpha\left(r-r^{\prime}\right) \sigma_{i j}^{(\mathrm{cl})}\left(r^{\prime}\right) \mathrm{d} v\left(r^{\prime}\right), \\
& \mu_{i j}(r)=\int_{V} \alpha\left(r-r^{\prime}\right) \mu_{i j}^{(\mathrm{cl})}\left(r^{\prime}\right) \mathrm{d} v\left(r^{\prime}\right) .
\end{aligned}
$$

Using the nonlocal kernel (2.30) and applying the Helmholtz operator to (3.54) and (3.55), one obtains

$$
\begin{aligned}
& \left(1-\varepsilon^{2} \Delta\right) \sigma_{i j}=\sigma_{i j}^{(\mathrm{cl})} \\
& \left(1-\varepsilon^{2} \Delta\right) \mu_{i j}=\mu_{i j}^{(\mathrm{cl})},
\end{aligned}
$$

where $\sigma_{i j}^{(\mathrm{cl})}$ and $\mu_{i j}^{(\mathrm{cl})}$ are the classical local stress and couple stress tensors given by

$$
\begin{aligned}
& \sigma_{i j}^{(\mathrm{cl})}=\lambda \delta_{i j} \gamma_{k k}^{(\mathrm{cl})}+(\mu+\eta) \gamma_{i j}^{(\mathrm{cl})}+(\mu-\eta) \gamma_{j i}^{(\mathrm{cl})} \\
& \mu_{i j}^{(\mathrm{cl})}=\alpha \delta_{i j} \kappa_{k k}^{(\mathrm{cl})}+(\beta+\gamma) \kappa_{i j}^{(\mathrm{cl})}+(\beta-\gamma) \kappa_{j i}^{(\mathrm{cl})}
\end{aligned}
$$

Here $\gamma_{i j}^{(\mathrm{cl})}$ and $\gamma_{i j}^{(\mathrm{cl})}$ are the local micropolar distortion and micropolar bend-twist tensors, respectively. It is important to note that the local micropolar material constants in Eqs. (3.58) and (3.59) are the same which appear in (3.20) and (3.21).

Again if we identify $\sigma_{i j}^{(\mathrm{cl})}=\stackrel{\circ}{\sigma}_{i j}$ and $\mu_{i j}^{(\mathrm{cl})}=\stackrel{\circ}{\mu}_{i j}$, Eqs. (3.37) and (3.38) coincide with (3.56) and (3.57), respectively. In addition the stresses fulfill the equilibrium conditions (3.52) and (3.53) in gradient micropolar elasticity, too. No double force and double couple stresses appear in nonlocal micropolar elasticity. 


\subsection{Screw Dislocation}

The expressions for the force stresses and couple stresses of a screw dislocations in a Cosserat continuum or micropolar medium were given by Kessel [41] (see also [39, 40,42, 43]). In micropolar elasticity, the asymmetric force stresses of a straight screw dislocation may be given in terms of the stress function

$$
\stackrel{\circ}{\sigma}_{z \varphi}=\partial_{r} \stackrel{\circ}{F}_{-}, \quad \stackrel{\circ}{\sigma}_{\varphi z}=\partial_{r} \stackrel{\circ}{F}_{+},
$$

where the two stress functions $\stackrel{\circ}{F}_{ \pm}$are given by

$$
\stackrel{\circ}{F}_{ \pm}=\frac{b_{z}}{2 \pi}\left\{\mu \ln r \pm \eta K_{0}(r / h)\right\} .
$$

They fulfill

$$
\left(1-h^{2} \Delta\right) \Delta \stackrel{\circ}{F}_{ \pm}=b_{z}\left\{(\mu \mp \eta)\left[1-h^{2} \Delta\right] \pm \eta\right\} \delta(x) \delta(y) .
$$

In gradient micropolar elasticity, the asymmetric force stress can be expressed in terms of new stress functions $F_{-}$and $F_{+}$:

$$
\sigma_{z \varphi}=\partial_{r} F_{-}, \quad \sigma_{\varphi z}=\partial_{r} F_{+} .
$$

If we substitute (3.60), (3.61) and (3.63) into the Helmholtz equation for the force stress tensor (3.37), we obtain for the modified stress functions the following inhomogeneous Helmholtz equation

$$
\left(1-\varepsilon^{2} \Delta\right) F_{ \pm}=\frac{b_{z}}{2 \pi}\left\{\mu \ln r \pm \eta K_{0}(r / h)\right\}
$$

where the inhomogeneous part is given by the stress functions (3.61). The nonsingular solution of (3.64) is [31]

$$
F_{ \pm}=\frac{b_{z}}{2 \pi}\left\{\mu\left[\ln r+K_{0}(r / \varepsilon)\right] \pm \eta \frac{h^{2}}{h^{2}-\varepsilon^{2}}\left[K_{0}(r / h)-K_{0}(r / \varepsilon)\right]\right\} .
$$

It is a more complicated superposition of the micropolar stress function (3.61) and a gradient coefficient depending term that in strain gradient elasticity ${ }^{1}$. In addition, they satisfy

$$
\left(1-\varepsilon^{2} \Delta\right)\left(1-h^{2} \Delta\right) \Delta F_{ \pm}=b_{z}\left\{(\mu \mp \eta)\left[1-h^{2} \Delta\right] \pm \eta\right\} \delta(x) \delta(y) .
$$

Using (3.63) and (3.65), the asymmetric force stress is found as

$$
\begin{aligned}
& \sigma_{z \varphi}=\frac{b_{z}}{2 \pi} \frac{1}{r}\left\{\mu\left[1-\frac{r}{\varepsilon} K_{1}(r / \varepsilon)\right]+\frac{\eta h^{2}}{h^{2}-\varepsilon^{2}}\left[\frac{r}{h} K_{1}(r / h)-\frac{r}{\varepsilon} K_{1}(r / \varepsilon)\right]\right\}, \\
& \sigma_{\varphi z}=\frac{b_{z}}{2 \pi} \frac{1}{r}\left\{\mu\left[1-\frac{r}{\varepsilon} K_{1}(r / \varepsilon)\right]-\frac{\eta h^{2}}{h^{2}-\varepsilon^{2}}\left[\frac{r}{h} K_{1}(r / h)-\frac{r}{\varepsilon} K_{1}(r / \varepsilon)\right]\right\} .
\end{aligned}
$$

They are zero at $r=0$ and have extremum values near the dislocation line. The extremum values depend on $h$ and $\varepsilon$. For instance, with $h=2 \varepsilon$ and $\mu=3 \eta$ we obtain: $\sigma_{z \varphi} \simeq 0.499 \mu b_{z} /[2 \pi \varepsilon]$ at $r \simeq 1.024 \varepsilon$ and $\sigma_{\varphi z} \simeq 0.303 \mu b_{z} /[2 \pi \varepsilon]$ at $r \simeq 1.312 \varepsilon$. Thus, $\sigma_{z \varphi}>\sigma_{\varphi z}$. The stresses are plotted versus $r / \varepsilon$ in Fig. 5. We note that the stresses (3.67) and (3.68) are also solutions of Eq. (3.52) in nonlocal micropolar elasticity. In nonlocal micropolar elasticity these solutions

\footnotetext{
${ }^{1} \operatorname{In}[31,32]$ we used the notation $\kappa=1 / \varepsilon, \tau=1 / h$ and $\zeta=1 / l$. In the current notation the pre-factors and the limits to micropolar elasticity are easier to handle.
} 

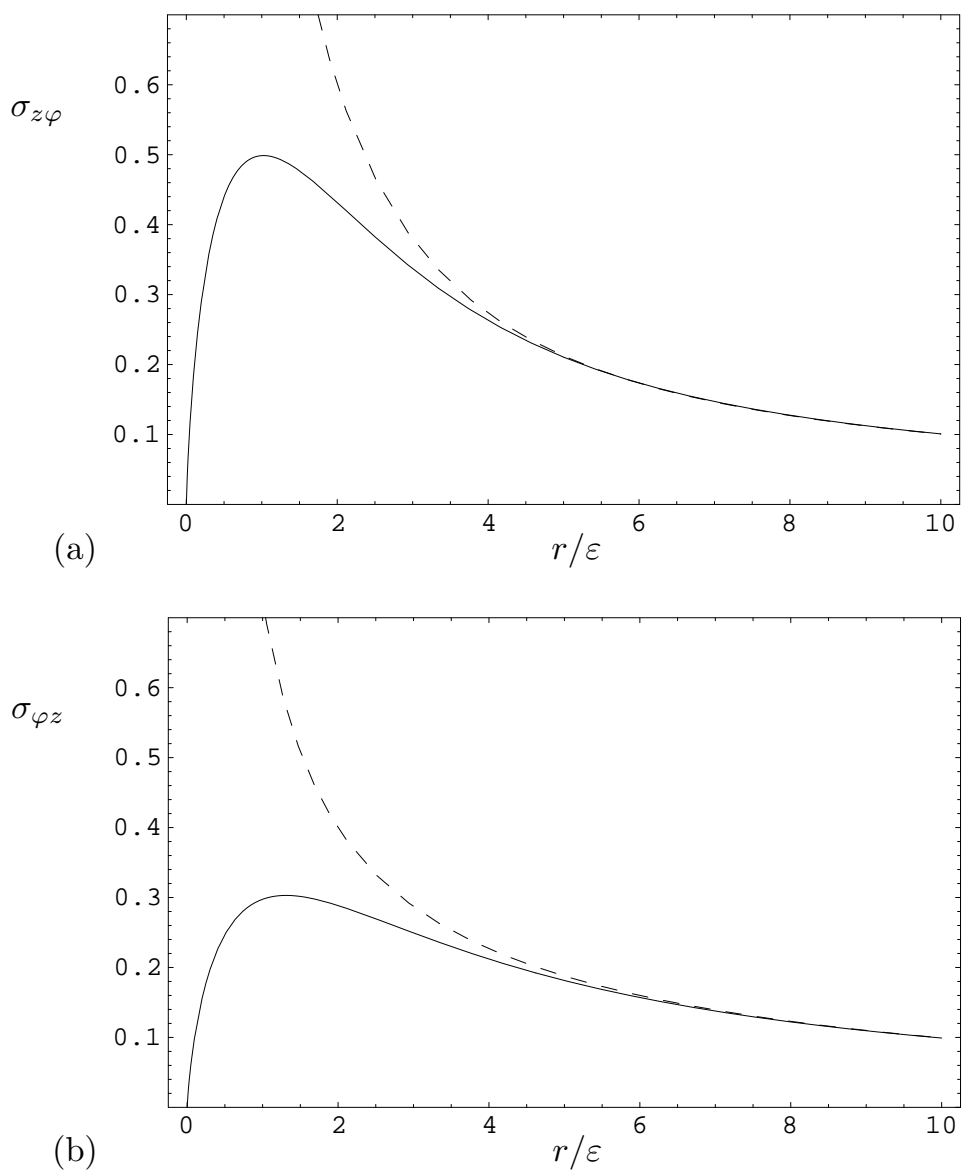

Figure 5: The components of the asymmetric stress of a screw dislocation: (a) $\sigma_{z \varphi}$ and (b) $\sigma_{\varphi z}$ are given in units of $\mu b_{z} /[2 \pi \varepsilon]$ with $h=2 \varepsilon$ and $\mu=3 \eta$. The dashed curves represent the micropolar results.

were calculated by Povstenko [29] (He used the transposed tensors.). In addition, in the limit to micropolar elasticity, $\varepsilon \rightarrow 0$, we recover in (3.67) and (3.68) Kessel's result [41].

By means of the inverse of the constitutive relation (3.20), the micropolar distortion is obtained as

$$
\begin{aligned}
& \gamma_{z \varphi}=\frac{b_{z}}{4 \pi} \frac{1}{r}\left\{\left[1-\frac{r}{\varepsilon} K_{1}(r / \varepsilon)\right]+\frac{h^{2}}{h^{2}-\varepsilon^{2}}\left[\frac{r}{h} K_{1}(r / h)-\frac{r}{\varepsilon} K_{1}(r / \varepsilon)\right]\right\}, \\
& \gamma_{\varphi z}=\frac{b_{z}}{4 \pi} \frac{1}{r}\left\{\left[1-\frac{r}{\varepsilon} K_{1}(r / \varepsilon)\right]-\frac{h^{2}}{h^{2}-\varepsilon^{2}}\left[\frac{r}{h} K_{1}(r / h)-\frac{r}{\varepsilon} K_{1}(r / \varepsilon)\right]\right\} .
\end{aligned}
$$

They are zero at $r=0$ and have extremum values near the dislocation line. Again, the extremum values depend on $h$ and $\varepsilon$. For instance, with $h=2 \varepsilon$ we have: $\gamma_{z \varphi} \simeq 0.701 b_{z} /[4 \pi \varepsilon]$ at $r \simeq 0.941 \varepsilon$ and $\gamma_{\varphi z} \simeq 0.163 b_{z} /[4 \pi \varepsilon]$ at $r \simeq 3.395 \varepsilon$. Thus, it yields $\gamma_{z \varphi}>\gamma_{\varphi z}$. In addition, $\stackrel{\circ}{\gamma}_{\varphi z} \simeq 0.151 b_{z} /[4 \pi \varepsilon]$ at $r \simeq 2.229 \varepsilon$. The micropolar distortions (3.69) and (3.70) are plotted in Fig. 6 versus $r / \varepsilon$ and with $h=2 \varepsilon$. It is interesting to note that the elastic micropolar strain $\gamma_{(z \varphi)}$ has the same form as the elastic strain $E_{z \varphi}$ calculated in gradient elasticity (see Eq. 2.42). In the limit $\varepsilon \rightarrow 0$, we recover in (3.69) and (3.70) Minagawa's result [42]. 

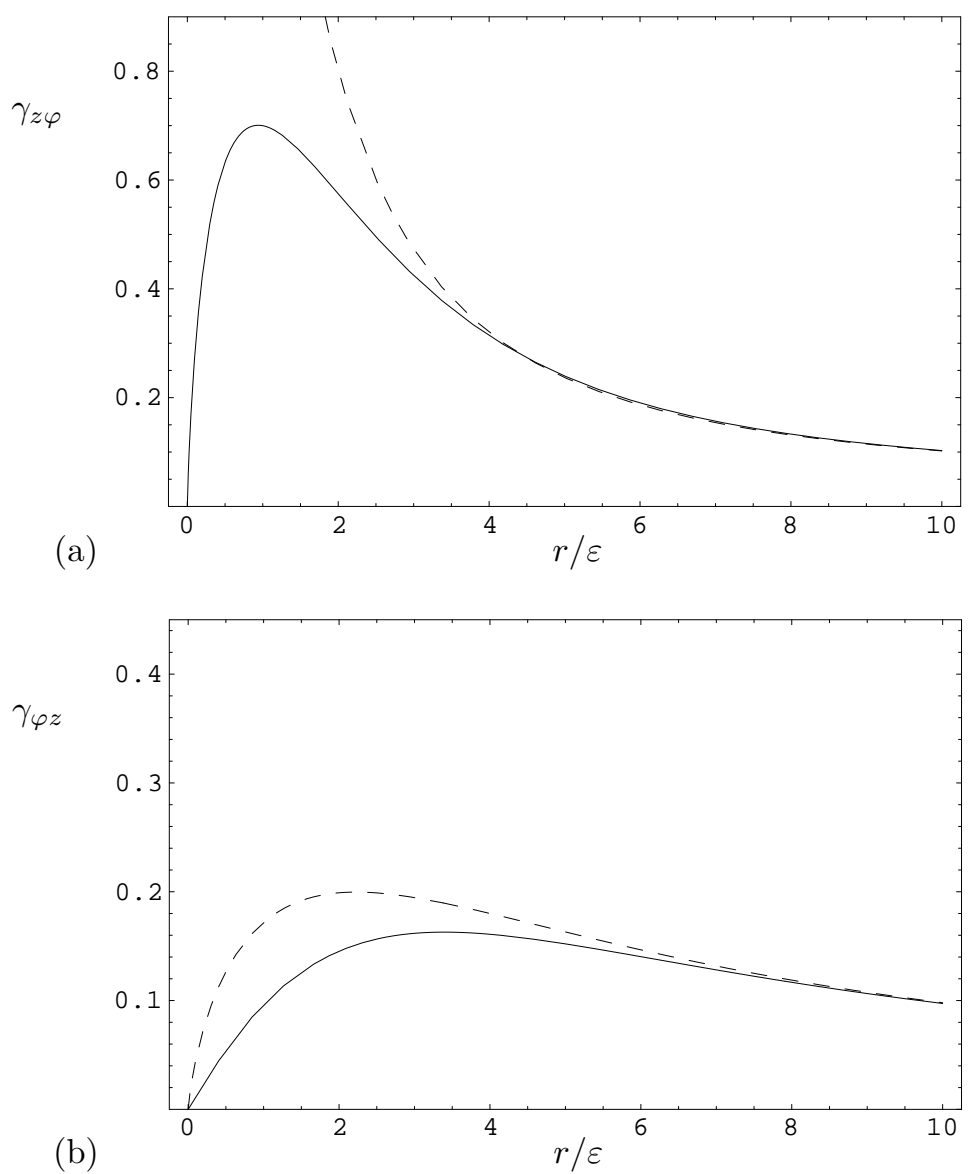

Figure 6: The components of the micropolar distortion for a screw dislocation: (a) $\gamma_{z \varphi}$ and (b) $\gamma_{\varphi z}$ are given in units of $b_{z} /[4 \pi \varepsilon]$ and $h=2 \varepsilon$. The dashed curves represent the micropolar results.

The micro-rotation of a straight screw dislocation reads [31]

$$
\varphi_{r}=\gamma_{\varphi z}=\frac{1}{2} \partial_{r} F_{+}(\mu=1, \eta=1)
$$

On the other hand, $\varphi_{r}$ follows directly from Eq. (3.46) with $\stackrel{\circ}{\varphi}_{r}=\stackrel{\circ}{\varphi}_{\varphi z}$. The micropolar bendtwist or wryness is given in terms of (3.71)

$$
\kappa_{r r}=\partial_{r} \varphi_{r}, \quad \kappa_{\varphi \varphi}=\frac{1}{r} \varphi_{r} .
$$

So, we find

$$
\begin{aligned}
& \kappa_{r r}=-\frac{b_{z}}{4 \pi} \frac{1}{r^{2}}\left\{1-\frac{1}{h^{2}-\varepsilon^{2}}\left[h r K_{1}(r / h)-\varepsilon r K_{1}(r / \varepsilon)+r^{2}\left(K_{0}(r / h)-K_{0}(r / \varepsilon)\right)\right]\right\}, \\
& \kappa_{\varphi \varphi}=\frac{b_{z}}{4 \pi} \frac{1}{r^{2}}\left\{1-\frac{1}{h^{2}-\varepsilon^{2}}\left[h r K_{1}(r / h)-\varepsilon r K_{1}(r / \varepsilon)\right]\right\} .
\end{aligned}
$$

The trace of the micropolar bend-twist tensor reads

$$
\kappa_{k k}=\frac{b_{z}}{4 \pi} \frac{1}{h^{2}-\varepsilon^{2}}\left[K_{0}(r / h)-K_{0}(r / \varepsilon)\right] .
$$


The components $\kappa_{r r}, \kappa_{\varphi \varphi}$ and $\kappa_{k k}$ have a maximum at the dislocation line. In fact, one obtains at $r=0$ :

$$
\kappa_{r r}=\kappa_{\varphi \varphi}=\frac{1}{2} \kappa_{k k} \simeq \frac{b_{z}}{4 \pi} \frac{1}{2\left(h^{2}-\varepsilon^{2}\right)} \ln \frac{h}{\varepsilon} .
$$

For the choice $h=2 \varepsilon$ we have at $r=0$ :

$$
\kappa_{r r}=\kappa_{\varphi \varphi}=\frac{1}{2} \kappa_{k k} \simeq 0.116 \frac{b_{z}}{4 \pi \varepsilon^{2}} .
$$

Thus, all $1 / r^{2}$ - and $\ln r$-singularities, which are present in the results of Cosserat theory, are eliminated. In addition, the bend-twist is modified in the region $r / \varepsilon<6$. The shape of the bend-twist in gradient micropolar elasticity looks more physical than the bend-twist in classical micropolar elasticity (see Fig. 7). In addition, it can be seen that $\kappa_{r r}$ is negative in the far field expression and positive near the dislocation line. The point at which it changes sign is for $h=2 \varepsilon: r \simeq 3.395 \varepsilon$ (see Fig. 7a). In the limit $\varepsilon \rightarrow 0$, we recover in (3.73) and (3.74) Minagawa's result [42].

Using Eq. (3.21) one obtains from the micropolar bend-twist tensor the couple-stress tensor. The non-vanishing components of the micropolar couple stress tensor are

$$
\begin{aligned}
& \mu_{r r}=-\frac{b_{z}}{2 \pi} \frac{1}{r^{2}}\left\{\beta-\frac{1}{h^{2}-\varepsilon^{2}}\left[\beta\left(h r K_{1}(r / h)-\varepsilon r K_{1}(r / \varepsilon)\right)+\frac{\alpha+2 \beta}{2} r^{2}\left(K_{0}(r / h)-K_{0}(r / \varepsilon)\right)\right]\right\}, \\
& \mu_{\varphi \varphi}=\frac{b_{z}}{2 \pi} \frac{1}{r^{2}}\left\{\beta-\frac{1}{h^{2}-\varepsilon^{2}}\left[\beta\left(h r K_{1}(r / h)-\varepsilon r K_{1}(r / \varepsilon)\right)-\frac{\alpha}{2}\left(K_{0}(r / h)-K_{0}(r / \varepsilon)\right)\right]\right\}, \\
& \mu_{z z}=\frac{b_{z} \alpha}{4 \pi} \frac{1}{h^{2}-\varepsilon^{2}}\left[K_{0}(r / h)-K_{0}(r / \varepsilon)\right] .
\end{aligned}
$$

The trace of the couple stress tensor is given by

$$
\mu_{k k}=\frac{b_{z}(3 \alpha+2 \beta)}{4 \pi} \frac{1}{h^{2}-\varepsilon^{2}}\left[K_{0}(r / h)-K_{0}(r / \varepsilon)\right] .
$$

All components of the couple stress tensor are nonsingular in the gradient micropolar elasticity. In fact, one obtains at $r=0$ :

$$
\mu_{z z}=\frac{\alpha}{\beta+\alpha} \mu_{\varphi \varphi}=\frac{\alpha}{\beta+\alpha} \mu_{r r}=\frac{\alpha}{2 \beta+3 \alpha} \mu_{k k} \simeq \alpha \frac{b_{z}}{2 \pi} \frac{1}{2\left(h^{2}-\varepsilon^{2}\right)} \ln \frac{h}{\varepsilon} .
$$

The micropolar bend-twist and the couple stress tensors of a screw dislocation are symmetric tensors. The far-fields of (3.73) and (3.74), and (3.78)-(3.80) agree with the result given by Kessel [41] and Minagawa [42]. In the limit $\varepsilon \rightarrow 0$, we recover in (3.78)-(3.80) Kessel's result [41].

The couple stresses (3.78)-(3.80) are the solutions in nonlocal elasticity. They fulfill together with the stresses (3.67) and (3.68) the moment equilibrium (3.52) in nonlocal micropolar elasticity. Using the relation $K_{2}(x)-K_{0}(x)=2 / x K_{1}(x)$, it can be shown that the couple stresses agree with the couple stresses in nonlocal micropolar elasticity calculated by Povstenko [29] up to a pre-factor $1 / 2$ which is missing in his formulas. This is because he used Nowacki's expressions [39] for the couples stresses of a screw dislocation in Cosserat theory which are the source of this missing factor 1/2. The results of Kessel [41] and Minagawa [42] have the correct pre-factor. The components of the force and couple stress, distortion and bend-twist tensors in Cartesian coordinates are given in Ref. [31].

\subsection{Edge Dislocation}

The expressions of the force stresses and couple stresses of a straight edge dislocations in micropolar elasticity (Cosserat theory) are given by [39-41,44,45]. We use the stress function 

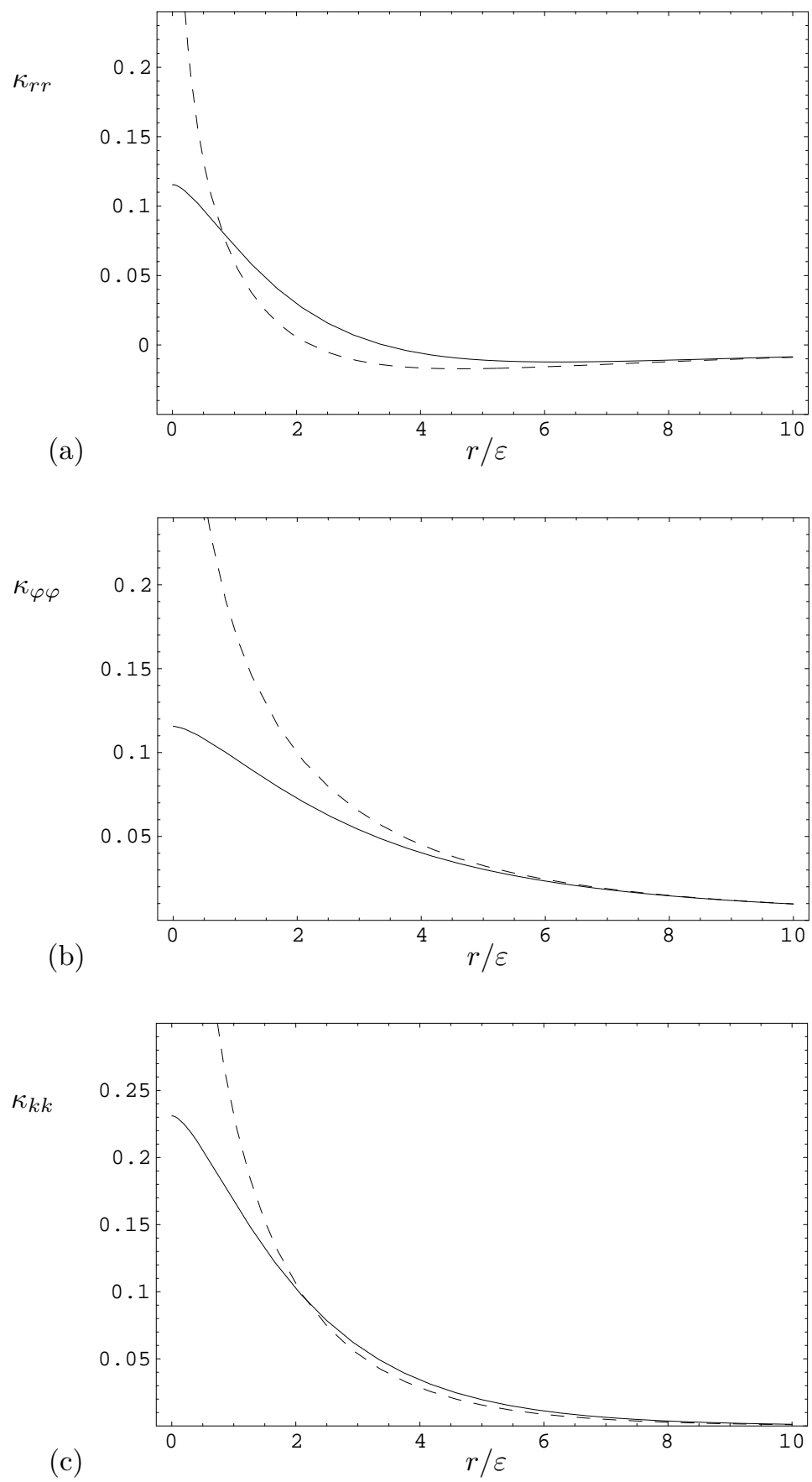

Figure 7: The components of the micropolar bend-twist tensor for a screw dislocation: (a) $\kappa_{r r}$, (b) $\kappa_{\varphi \varphi}$ and (c) $\kappa_{k k}$ are given in units of $b_{z} /\left[4 \pi \varepsilon^{2}\right]$ and with $h=2 \varepsilon$. The dashed curves represent the classical (micropolar) components. 
formulation given by $[46,47]$ (see also $[38,40,43]$ ), which involves two stress functions $\stackrel{\circ}{f}$ and $\stackrel{\circ}{\Psi}$, which are connected with the force stresses and couple stresses by

$$
\begin{aligned}
& \stackrel{\circ}{\sigma}_{r r}=\frac{1}{r} \partial_{r} \stackrel{\circ}{f}+\frac{1}{r^{2}} \partial_{\varphi \varphi}^{2} \stackrel{\circ}{f}-\frac{1}{r} \partial_{r \varphi}^{2} \stackrel{\circ}{\Psi}+\frac{1}{r^{2}} \partial_{\varphi} \stackrel{\circ}{\Psi}, \\
& \stackrel{\circ}{\sigma}_{\varphi \varphi}=\partial_{r r}^{2} \stackrel{\circ}{f}+\frac{1}{r} \partial_{r \varphi}^{2} \stackrel{\circ}{\Psi}-\frac{1}{r^{2}} \partial_{\varphi} \stackrel{\circ}{\Psi}, \\
& \stackrel{\circ}{\sigma}_{r \varphi}=-\frac{1}{r} \partial_{r \varphi}^{2} \stackrel{\circ}{f}+\frac{1}{r^{2}} \partial_{\varphi} \stackrel{\circ}{f}+\partial_{r r}^{2} \stackrel{\circ}{\Psi}, \\
& \stackrel{\circ}{\sigma}_{\varphi r}=-\frac{1}{r} \partial_{r \varphi}^{2} \stackrel{\circ}{f}+\frac{1}{r^{2}} \partial_{\varphi} \stackrel{\circ}{f}-\frac{1}{r} \partial_{r} \stackrel{\circ}{\Psi}-\frac{1}{r^{2}} \partial_{\varphi \varphi}^{2} \stackrel{\circ}{\Psi}, \\
& \stackrel{\circ}{\mu}_{z r}=\partial_{r} \stackrel{\circ}{\Psi}, \quad \stackrel{\circ}{\mu}_{z \varphi}=\frac{1}{r} \partial_{\varphi} \stackrel{\circ}{\Psi}
\end{aligned}
$$

and $\stackrel{\circ}{\sigma}_{z z}=\nu\left(\stackrel{\circ}{\sigma}_{r r}+\stackrel{\circ}{\sigma}_{\varphi \varphi}\right)$. Here $\stackrel{\circ}{f}$ is the Airy stress function known from classical elasticity and $\stackrel{\circ}{\Psi}$ is the stress function introduced by Mindlin [46], Schaefer [47] and Carlson [48]. For a straight edge dislocation the so-called Airy-Mindlin stress functions reads [39, 40]

$$
\begin{aligned}
& \stackrel{\circ}{f}=-\frac{\mu b_{x}}{2 \pi(1-\nu)} \sin \varphi(r \ln r), \\
& \stackrel{\circ}{\Psi}=-\frac{(\beta+\gamma) b_{x}}{2 \pi} \frac{\cos \varphi}{r}\left(1-\frac{r}{l} K_{1}(r / l)\right)
\end{aligned}
$$

with

$$
\left(1-l^{2} \Delta\right) \stackrel{\circ}{\Psi}=-\frac{(\beta+\gamma) b_{x}}{2 \pi} \frac{\cos \varphi}{r} .
$$

With the help of Eq. (3.90) and some simple manipulations, we find the Cauchy-Riemann relations

$$
\begin{aligned}
\partial_{r}\left(1-l^{2} \Delta\right) \stackrel{\circ}{\Psi} & =-\frac{2(1-\nu)}{\mu(\beta+\gamma)} \frac{1}{r} \partial_{\varphi}(\Delta \stackrel{\circ}{f}), \\
\frac{1}{r} \partial_{\varphi}\left(1-l^{2} \Delta\right) \stackrel{\circ}{\Psi} & =\frac{2(1-\nu)}{\mu(\beta+\gamma)} \partial_{r}(\Delta \stackrel{\circ}{f}) .
\end{aligned}
$$

By construction this stress function ansatz fulfills the equilibrium conditions (3.52) and (3.53). All components of the force stress tensor have $1 / r$-singularities and those of the couple stress tensor have $1 / r^{2}$-singularities.

In gradient micropolar elasticity we make the following ansatz

$$
\begin{aligned}
\sigma_{r r} & =\frac{1}{r} \partial_{r} f+\frac{1}{r^{2}} \partial_{\varphi \varphi}^{2} f-\frac{1}{r} \partial_{r \varphi}^{2} \Psi+\frac{1}{r^{2}} \partial_{\varphi} \Psi, \\
\sigma_{\varphi \varphi} & =\partial_{r r}^{2} f+\frac{1}{r} \partial_{r \varphi}^{2} \Psi-\frac{1}{r^{2}} \partial_{\varphi} \Psi, \\
\sigma_{r \varphi} & =-\frac{1}{r} \partial_{r \varphi}^{2} f+\frac{1}{r^{2}} \partial_{\varphi} f+\partial_{r r}^{2} \Psi, \\
\sigma_{\varphi r} & =-\frac{1}{r} \partial_{r \varphi}^{2} f+\frac{1}{r^{2}} \partial_{\varphi} f-\frac{1}{r} \partial_{r} \Psi-\frac{1}{r^{2}} \partial_{\varphi \varphi}^{2} \Psi, \\
\mu_{z r} & =\partial_{r} \Psi, \quad \mu_{z \varphi}=\frac{1}{r} \partial_{\varphi} \Psi
\end{aligned}
$$

and $\sigma_{z z}=\nu\left(\sigma_{r r}+\sigma_{\varphi \varphi}\right)$. It has the same form as the stress function ansatz in micropolar elasticity. Here $f$ and $\Psi$ are the modified stress functions which must be calculated. So, we 
obtain for the modified stress functions the following inhomogeneous Helmholtz equations

$$
\begin{aligned}
& \left(1-\varepsilon^{2} \Delta\right) f=-\frac{\mu b_{x}}{2 \pi(1-\nu)} \sin \varphi(r \ln r), \\
& \left(1-\varepsilon^{2} \Delta\right) \Psi=-\frac{(\beta+\gamma) b_{x}}{2 \pi} \frac{\cos \varphi}{r}\left(1-\frac{r}{l} K_{1}(r / l)\right) .
\end{aligned}
$$

The nonsingular solutions are (see $[31,32]$ for technical details)

$$
\begin{aligned}
& f=-\frac{\mu b_{x}}{2 \pi(1-\nu)} \sin \varphi\left\{r \ln r+\frac{2 \varepsilon^{2}}{r}\left(1-\frac{r}{\varepsilon} K_{1}(r / \varepsilon)\right)\right\}, \\
& \Psi=-\frac{(\beta+\gamma) b_{x}}{2 \pi} \frac{\cos \varphi}{r}\left\{1-\frac{1}{l^{2}-\varepsilon^{2}}\left[\frac{r}{l} K_{1}(r / l)-\frac{r}{\varepsilon} K_{1}(r / \varepsilon)\right]\right\} .
\end{aligned}
$$

Again, they are superpositions of the classical stress functions (3.88) and (3.89) and the gradient parts which depend on the gradient coefficient $\varepsilon$. Therefore, the stress function (3.100) has the same form as in strain gradient elasticity (see Eq. (2.45)). The stress function (3.101) also satisfies the following bi-Helmholtz equation:

$$
\left(1-l^{2} \Delta\right)\left(1-\varepsilon^{2} \Delta\right) \Psi=-(\beta+\gamma) b_{x} \partial_{x} \delta(x) \delta(y) .
$$

Thus, it is the corresponding Green function. In addition, the stress functions $\Psi$ and $f$ are related by the following Cauchy-Riemann relations

$$
\begin{aligned}
\partial_{r}\left(1-\varepsilon^{2} \Delta\right)\left(1-l^{2} \Delta\right) \Psi & =-\frac{2(1-\nu)}{\mu(\beta+\gamma)} \frac{1}{r} \partial_{\varphi}\left(1-\varepsilon^{2} \Delta\right) \Delta f, \\
\frac{1}{r} \partial_{\varphi}\left(1-\varepsilon^{2} \Delta\right)\left(1-l^{2} \Delta\right) \Psi & =\frac{2(1-\nu)}{\mu(\beta+\gamma)} \partial_{r}\left(1-\varepsilon^{2} \Delta\right) \Delta f .
\end{aligned}
$$

Using the Airy-Mindlin stress functions (3.100) and (3.101), we find for the asymmetric force stress of a straight edge dislocation

$$
\begin{aligned}
\sigma_{r r}= & -\frac{\mu b_{x}}{2 \pi(1-\nu)} \frac{\sin \varphi}{r}\left\{1-\frac{4 \varepsilon^{2}}{r^{2}}+2 K_{2}(r / \varepsilon)\right\} \\
& +\frac{(\beta+\gamma) b_{x}}{2 \pi} \frac{\sin \varphi}{r^{3}}\left\{2-\frac{r^{2}}{l^{2}-\varepsilon^{2}}\left[K_{2}(r / l)-K_{2}(r / \varepsilon)\right]\right\} \\
\sigma_{\varphi \varphi}= & -\frac{\mu b_{x}}{2 \pi(1-\nu)} \frac{\sin \varphi}{r}\left\{1+\frac{4 \varepsilon^{2}}{r^{2}}-2 K_{2}(r / \varepsilon)-2 \frac{r}{\varepsilon} K_{1}(r / \varepsilon)\right\} \\
& -\frac{(\beta+\gamma) b_{x}}{2 \pi} \frac{\sin \varphi}{r^{3}}\left\{2-\frac{r^{2}}{l^{2}-\varepsilon^{2}}\left[K_{2}(r / l)-K_{2}(r / \varepsilon)\right]\right\} \\
\sigma_{r \varphi}= & \frac{\mu b_{x}}{2 \pi(1-\nu)} \frac{\cos \varphi}{r}\left\{1-\frac{4 \varepsilon^{2}}{r^{2}}+2 K_{2}(r / \varepsilon)\right\} \\
& -\frac{(\beta+\gamma) b_{x}}{2 \pi} \frac{\cos \varphi}{r^{3}}\left\{2-\frac{r^{2}}{l^{2}-\varepsilon^{2}}\left[K_{2}(r / l)-K_{2}(r / \varepsilon)+\frac{r}{l} K_{1}(r / l)-\frac{r}{\varepsilon} K_{1}(r / \varepsilon)\right]\right\} \\
\sigma_{\varphi r}= & \frac{\mu b_{x}}{2 \pi(1-\nu)} \frac{\cos \varphi}{r}\left\{1-\frac{4 \varepsilon^{2}}{r^{2}}+2 K_{2}(r / \varepsilon)\right\} \\
& -\frac{(\beta+\gamma) b_{x}}{2 \pi} \frac{\cos \varphi}{r^{3}}\left\{2-\frac{r^{2}}{l^{2}-\varepsilon^{2}}\left[K_{2}(r / l)-K_{2}(r / \varepsilon)\right]\right\} \\
\sigma_{z z}= & -\frac{\mu b_{x} \nu}{\pi(1-\nu)} \frac{\sin \varphi}{r}\left\{1-\frac{r}{\varepsilon} K_{1}(r / \varepsilon)\right\}
\end{aligned}
$$


and

$$
\sigma_{k k}=-\frac{\mu b_{x}(1+\nu)}{\pi(1-\nu)} \frac{\sin \varphi}{r}\left\{1-\frac{r}{\varepsilon} K_{1}(r / \varepsilon)\right\} .
$$

The stress fields have no artificial singularities at the dislocation line and the extremum stress occurs at a short distance away from the dislocation line (see Figs. 8). The stresses (3.109) and (3.110) are not influenced by the Cosserat constants and, thus, they have the same form as the corresponding expressions (2.52) and (2.53) in strain gradient elasticity. In Eqs. (3.105)-(3.108) the first parts agree with the stresses $(2.49)-(2.51)$ in strain gradient elasticity and the other parts are modified stresses due to the gradient micropolar elasticity. Near the dislocation line one can see the difference between the stresses calculated in strain gradient elasticity and in gradient micropolar elasticity. The extremum values of these stresses are changed. They are higher or lower than the extremum values in strain gradient theory (see Fig. 8). In addition, the values and the positions of the extremum values depend strongly on the material constants of gradient micropolar elasticity.

Again, the stresses (3.105)-(3.109) are solutions of Eq. (3.52) in nonlocal micropolar elasticity. This nonlocal solution coincides with the solution given by Povstenko and Matkovskii [30]. In the limit $\varepsilon \rightarrow 0$, we recover in (3.105)-(3.109) the micropolar result given by [39,41].

Using (3.26), we obtain the elastic micropolar distortion from the asymmetric force stress tensor

$$
\begin{aligned}
\gamma_{r r}= & -\frac{b_{x}}{4 \pi(1-\nu)} \frac{\sin \varphi}{r}\left\{(1-2 \nu)-\frac{4 \varepsilon^{2}}{r^{2}}+2 K_{2}(r / \varepsilon)+2 \nu \frac{r}{\varepsilon} K_{1}(r / \varepsilon)\right\} \\
& +\frac{(\beta+\gamma) b_{x}}{4 \pi \mu} \frac{\sin \varphi}{r^{3}}\left\{2-\frac{r^{2}}{l^{2}-\varepsilon^{2}}\left[K_{2}(r / l)-K_{2}(r / \varepsilon)\right]\right\}, \\
\gamma_{\varphi \varphi}= & -\frac{b_{x}}{4 \pi(1-\nu)} \frac{\sin \varphi}{r}\left\{(1-2 \nu)+\frac{4 \varepsilon^{2}}{r^{2}}-2 K_{2}(r / \varepsilon)-2(1-\nu) \frac{r}{\varepsilon} K_{1}(r / \varepsilon)\right\} \\
& -\frac{(\beta+\gamma) b_{x}}{4 \pi \mu} \frac{\sin \varphi}{r^{3}}\left\{2-\frac{r^{2}}{l^{2}-\varepsilon^{2}}\left[K_{2}(r / l)-K_{2}(r / \varepsilon)\right]\right\}, \\
\gamma_{r \varphi}= & \frac{b_{x}}{4 \pi(1-\nu)} \frac{\cos \varphi}{r}\left\{1-\frac{4 \varepsilon^{2}}{r^{2}}+2 K_{2}(r / \varepsilon)\right\} \\
& -\frac{(\beta+\gamma) b_{x}}{4 \pi \mu} \frac{\cos \varphi}{r^{3}}\left\{2-\frac{r^{2}}{l^{2}-\varepsilon^{2}}\left[K_{2}(r / l)-K_{2}(r / \varepsilon)+\frac{\eta+\mu}{2 \eta}\left(\frac{r}{l} K_{1}(r / l)-\frac{r}{\varepsilon} K_{1}(r / \varepsilon)\right)\right]\right\}, \\
\gamma_{\varphi r}= & \frac{b_{x}}{4 \pi(1-\nu)} \frac{\cos \varphi}{r}\left\{1-\frac{4 \varepsilon^{2}}{r^{2}}+2 K_{2}(r / \varepsilon)\right\} \\
& -\frac{(\beta+\gamma) b_{x}}{4 \pi \mu} \frac{\cos \varphi}{r^{3}}\left\{2-\frac{r^{2}}{l^{2}-\varepsilon^{2}}\left[K_{2}(r / l)-K_{2}(r / \varepsilon)+\frac{\eta-\mu}{2 \eta}\left(\frac{r}{l} K_{1}(r / l)-\frac{r}{\varepsilon} K_{1}(r / \varepsilon)\right)\right]\right\}
\end{aligned}
$$

and

$$
\gamma_{k k}=-\frac{\mu b_{x}(1-2 \nu)}{2 \pi(1-\nu)} \frac{\sin \varphi}{r}\left\{1-\frac{r}{\varepsilon} K_{1}(r / \varepsilon)\right\} .
$$

It can be seen that the elastic micropolar strain $\gamma_{(i j)}$ is influenced by the Cosserat constants $\beta$, $\gamma$ and $l$ and, therefore, it is different from the elastic strain (2.54)-(2.56) of an edge dislocation in strain gradient elasticity. Only the first parts of Eqs. (3.111)-(3.114) agree with the elastic strains (2.54)-(2.56) in strain gradient elasticity. The other parts of Eqs. (3.111)-(3.114) are modified stresses due to the gradient micropolar elasticity. On the other hand, the micropolar dilatation (3.115) has the same form as the dilatation (2.57) in strain gradient elasticity. In the limit $\varepsilon \rightarrow 0$, we recover in (3.111)-(3.114) the micropolar result given by [44]. 

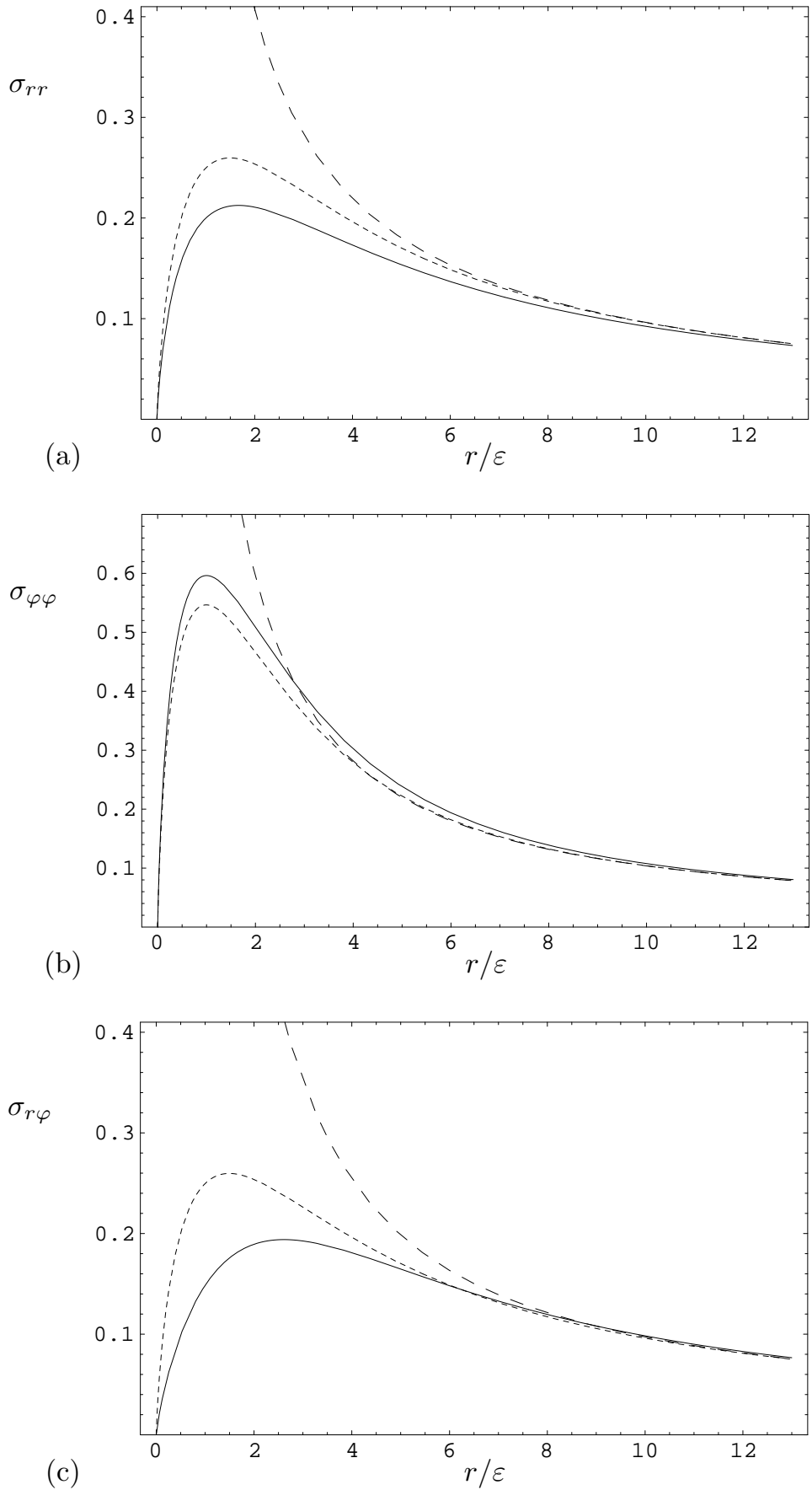

Figure 8: The components of stress: (a) $\sigma_{r r}$ and (b) $\sigma_{\varphi \varphi}$ are given in units of $\mu b_{z} /[2 \pi(1-\nu) \varepsilon]$ for $\varphi=3 \pi / 2$ and (c) $\sigma_{r \varphi}$ is given in units of $\mu b_{z} \nu /[\pi(1-\nu) \varepsilon]$ for $\varphi=0$. with $(\beta+\gamma)(1-\nu) / \mu=2$ and $l=2 \varepsilon$. The dashed curves represent the stresses in micropolar elasticity and the small dashed curves the stresses in strain gradient elasticity. 
Using Eqs. (3.97) and (3.101), we find for the micropolar couple stresses

$$
\begin{aligned}
& \mu_{z r}=\frac{(\beta+\gamma) b_{x}}{2 \pi} \frac{\cos \varphi}{r^{2}}\left\{1-\frac{1}{l^{2}-\varepsilon^{2}}\left[\operatorname{lr} K_{1}(r / l)-\varepsilon r K_{1}(r / \varepsilon)+r^{2}\left(K_{0}(r / l)-K_{0}(r / \varepsilon)\right)\right]\right\} \\
& \mu_{z \varphi}=\frac{(\beta+\gamma) b_{x}}{2 \pi} \frac{\sin \varphi}{r^{2}}\left\{1-\frac{1}{l^{2}-\varepsilon^{2}}\left[\operatorname{lr} K_{1}(r / l)-\varepsilon r K_{1}(r / \varepsilon)\right]\right\} \\
& \mu_{r z}=\frac{\beta-\gamma}{\beta+\gamma} \mu_{z r} \\
& \mu_{\varphi z}=\frac{\beta-\gamma}{\beta+\gamma} \mu_{z \varphi}
\end{aligned}
$$

Due to the special choice of cylindrical coordinates the couple stresses (3.116)-(3.119) have an artifical discontinuity at $r=0$. At $r=0$ the radial part of the couple stresses is finite and is multiplied by $\cos \varphi$ or $\sin \varphi$. In Cartesian coordinates the discontinuity disappears (see [32]). The couple stresses (3.116)-(3.119) are in agreement with the nonlocal couple stresses calculated by Povstenko and Matkovskii [30]. In the limit $\varepsilon \rightarrow 0$, we recover in (3.116)-(3.119) the micropolar result given by [41].

With (3.26) we obtain from the micropolar couple stresses the corresponding micropolar bend-twist (wryness)

$$
\begin{aligned}
\kappa_{z r} & =\frac{b_{x}}{2 \pi} \frac{\cos \varphi}{r^{2}}\left\{1-\frac{1}{l^{2}-\varepsilon^{2}}\left[\operatorname{lr} K_{1}(r / l)-\varepsilon r K_{1}(r / \varepsilon)+r^{2}\left(K_{0}(r / l)-K_{0}(r / \varepsilon)\right)\right]\right\}, \\
\kappa_{z \varphi} & =\frac{b_{x}}{2 \pi} \frac{\sin \varphi}{r^{2}}\left\{1-\frac{1}{l^{2}-\varepsilon^{2}}\left[\operatorname{lr} K_{1}(r / l)-\varepsilon r K_{1}(r / \varepsilon)\right]\right\} .
\end{aligned}
$$

The micropolar rotation can be calculated from the micropolar bend-twist. For example, if we use

$$
\varphi_{z}=r \int \kappa_{z \varphi} \mathrm{d} \varphi
$$

we obtain

$$
\varphi_{z}=-\frac{b_{x}}{2 \pi} \frac{\cos \varphi}{r}\left\{1-\frac{1}{l^{2}-\varepsilon^{2}}\left[\operatorname{lr} K_{1}(r / l)-\varepsilon r K_{1}(r / \varepsilon)\right]\right\} .
$$

For $\varepsilon \rightarrow 0,(3.123)$ coincides with Nowacki's result [40] calculated in the theory of micropolar elasticity. The components of the force and couple stress, distortion and bend-twist tensors in Cartesian coordinates can be found in Ref. [32].

\section{Conclusion}

In this paper, we have given an overview over static theories of gradient elasticity and nonlocal elasticity of Helmholtz type. We have discussed the theory of defects (dislocations, disclinations) in (gradient) elasticity as well as (gradient) micropolar elasticity. Such theories are incompatible gradient theories because the elastic distortion quantities are not simple gradients of the displacement and the rotation. We have shown the equivalence between the nonsingular stresses of screw and edge dislocation in gradient elasticity and the corresponding nonlocal stresses. In addition, we have investigated the relationship between gradient micropolar elasticity and nonlocal micropolar elasticity of Helmholtz type. For the static case, we have proven the equivalence of the force stresses and couple stresses calculated in gradient micropolar elasticity and nonlocal micropolar elasticity. We have considered straight screw and edge dislocation. In order to fulfill the equilibrium conditions we have used the stress function method. These stress 
functions are Green's function of partial differential equations of higher order. The calculated force and couple stresses do not possess singularities in the dislocation core region. For the gradient theories we have calculated the elastic distortion and bend-twist tensors of screw and edge dislocations. Unlike the nonlocal theories, where these fields still possess singularities at the dislocation line, the quantities calculated in the gradient theories are nonsingular. All fields calculated in the theories of gradient elasticity or gradient micropolar elasticity have the correct limits to classical elasticity or to micropolar elasticity.

\section{Acknowledgement}

This work was supported by the European Network RTN "DEFINO" with contract number HPRN-CT-2002-00198.

\section{References}

[1] Kröner, E., Datta, B.K., 1966. Nichtlokale Elastostatik: Abteitung aus der Gittertheorie. Z. Physik 196, 203-211.

[2] Kröner, E, 1967. Elasticity theory of materials with long range cohesive forces, Int. J. Solids Struct. 3, 731-742.

[3] Eringen, A.C., Edelen, D.G.B., 1972. On nonlocal elasticity, Int. J. Engng. Sci. 10, 233248.

[4] Kunin, I.A., 1983. Elastic Media with Microstructure II: Three-Dimensional Models, Springer, Berlin.

[5] Eringen, A.C., 1977. Screw dislocation in nonlocal elasticity, J. Phys. D: Appl. Phys. 10, 671-678.

[6] Eringen, A.C., 1977. Edge dislocation in nonlocal elasticity, Int. J. Engng. Sci. 15, 177-183.

[7] Ari, N., Eringen, A.C., 1983. Nonlocal stress field at Griffith crack, Cryst. Latt. Def. and Amorph. Mat. 10, 33-38.

[8] Eringen, A.C., 1983. On differential equations of nonlocal elasticity and solutions of screw dislocation and surface waves. J. Appl. Phys. 54, 4703-4710.

[9] Eringen, A.C., 1983. Interaction of a dislocation with a crack. J. Appl. Phys. 54, 6811-6817.

[10] Eringen, E.C., 2002. Nonlocal Continuum Field Theories, Springer, New York.

[11] Povstenko, Yu.Z., 1995. Circular dislocation loops in nonlocal elasticity. J. Phys. D: Appl. Phys 28, 105-111.

[12] Povstenko, Yu.Z., 1995. Straight disclinations in nonlocal elasticity. Int. J. Engng. Sci. 33, $575-582$.

[13] Povstenko, Yu.Z., Matkovskii, O.A., 2000. Circular disclination loops in nonlocal elasticity. Int. J. Solids Structures 37, 6419-6432.

[14] Lazar, M., 2003. A nonsingular solution of the edge dislocation in the gauge theory of dislocations. J. Phys. A: Math. Gen. 36, 1415-1437.

[15] Kröner, E., 1963. On the physical reality of torque stresses in continuum mechanics. Int. J. Engng. Sci. 1, 261-278. 
[16] Mindlin, R.D., 1964. Micro-structure in linear elasticity. Arch. Rat. Mech. Anal. 16, 51-78.

[17] Mindlin, R.D., 1965. Second gradient of strain and surface-tension in linear elasticity. Int. J. Solids Structures 1, 417-438.

[18] Mindlin, R.D., Eshel, N.N., 1968. On first strain gradients theory in linear elasticity. Int. J. Solids Structures 4, 109-124.

[19] Green, A.E., Rivlin, R.S., 1964. Simple force and stress multipoles, Arch. Rat. Mech. Anal. 16, 325-353.

[20] Green, A.E., Rivlin, R.S., 1964. Multipolar continuum mechanics, Arch. Rat. Mech. Anal. 17, 113-147.

[21] Gutkin, M.Yu., Aifantis, E.C., 1999. Dislocations in gradient elasticity. Scripta Mater. 40, $559-566$.

[22] Gutkin, M.Yu., 2000. Nanoscopics of dislocations and disclinations in gradient elasticity. Rev. Adv. Mater. Sci. 1, 27-60.

[23] Lazar, M., Maugin, G.A., 2003. Nonsingular stress and strain fields of dislocations and disclinations in first strain gradient elasticity. Int. J. Engng. Sci., in press.

[24] Gutkin, M.Yu., Aifantis, E.C., 1996. Screw dislocation in gradient elasticity. Scripta Mater. 35, 1353-1358.

[25] Gutkin, M.Yu., Aifantis, E.C., 1997. Edge dislocation in gradient elasticity. Scripta Mater. 36, 129-135.

[26] Gutkin, M.Yu., Aifantis, E.C., 1999. Dislocations and disclinations in gradient elasticity. Phys. Stat. Sol. (b) 214, 245-284.

[27] Aifantis, E.C., 2003. Update on a class of gradient theories. Mechanics of Materials 35, $259-280$.

[28] Eringen, A.C., 1984. Plane waves in nonlocal micropolar elasticity. Int. J. Engng. Sci. 22, $1113-1121$.

[29] Povstenko, Yu.Z., 1998. Imperfections in non-local elasticity. J. Phys. IV France 8, Pr8309-316.

[30] Povstenko, Yu.Z., Matkovskii, O.A., 1997. Edge dislocation in nonlocal elasticity medium with couple stresses. Mat. Met. Fiz.-Mekh. Polya. 40, 98-102 (in Ukrainian).

[31] Lazar, M., Maugin, G.A., 2004. Defects in gradient micropolar elasticity: I. Screw dislocation. J. Mech. Phys. Solids 52, 2263-2284.

[32] Lazar, M., Maugin, G.A., 2004. Defects in gradient micropolar elasticity: II. Edge dislocation and wedge disclination. J. Mech. Phys. Solids 52, 2285-2307.

[33] Zubov, L.M., 1997. Nonlinear thoery of dislocations and disclinations in elastic bodies. Springer.

[34] Altan, B.C., Aifantis, E.C., 1997. On some aspects in the special theory of gradient elasticity. J. Mech. Behav. Mater. 8, 231-282.

[35] Kröner, E., 1981. Continuum Theory of Defects, in: Physics of Defects (Les Houches, Session 35), R. Balian et al., eds., North-Holland, Amsterdam, p. 215. 
[36] DeWit, R., 1973. Theory of disclinations IV. J. Res. Nat. Bur. Stand. (U.S.) 77A, 607-658.

[37] Lazar, M., 2003. Dislocations in the field theory of elastoplasticity. Comput. Mater. Sci. 28, $419-428$.

[38] Eringen, A.C., 1999. Microcontinuum Field Theories I: Foundations and Solids, Springer, New York.

[39] Nowacki, W., 1974. On discrete dislocations in micropolar elasticity. Arch. Mech. 26, 3-11.

[40] Nowacki, W., 1986. Theory of Asymmetric Elasticity. Pergamon Press, Oxford.

[41] Kessel, S., 1970. Spannungsfelder einer Schraubenversetzung und einer Stufenversetzung im Cosserat Kontinuum. Z. Ang. Math. Mech. 50, 547-553.

[42] Minagawa, S., 1977. Elastic fields of dislocations and disclinations in an isotropic micropolar continuum. Appl. Eng. Sci. Lett. 5, 85-94.

[43] Povstenko, Yu.Z., 1994. Stress functions for continua with couple stresses. J. Elast. 36, 99-116.

[44] Minagawa, S., 1979. Stress and couple-stress fields produced by circular dislocations in an isotropic elastic microploar continuum. Z. Ang. Math. Mech. 59, 307-315.

[45] Knésl, Z., Semela, F., 1972. The influence of couple-stresses on the elastic properties of an edge dislocation. Int. J. Engng. Sci. 10, 83-91.

[46] Mindlin, R.D., 1963. Influence of couple-stresses on stress concentrations. Exper. Mech. 3, $1-7$.

[47] Schaefer, H., 1962. Versuch einer Elastizitätstheorie des zweidimensionalen CosseratKontinuums. Misz. Angew. Math., Festschrift Tollmien, Berlin, 277-292.

[48] Carlson, D.E., 1966. Stress functions for plane problems with couple stresses. Z. Ang. Math. Phys. 17, 789-792. 


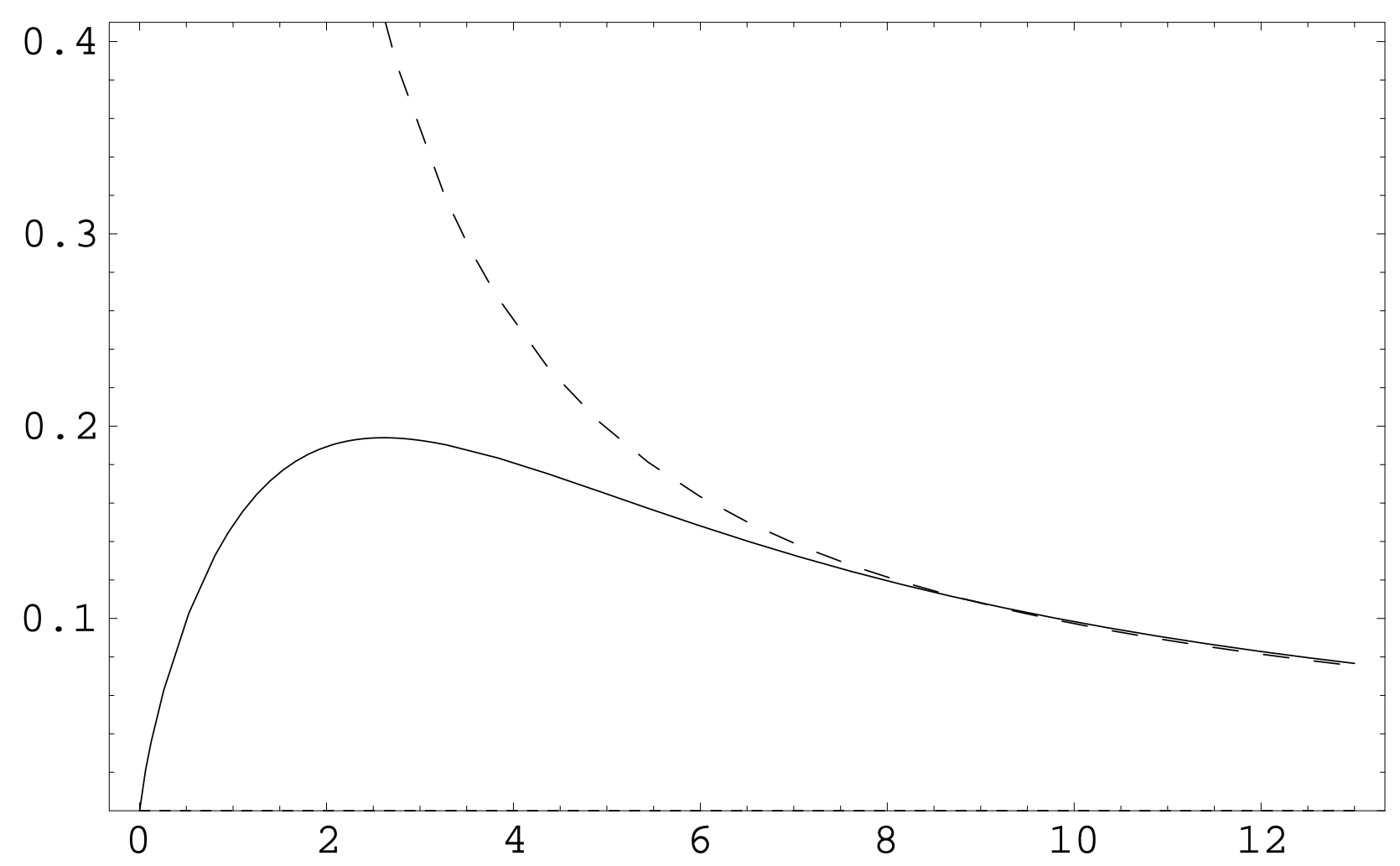

